\title{
Distributionally Robust Equilibrium for Continuous Games: Nash and Stackelberg Models
}

\author{
Yongchao $\mathrm{Liu}^{1}$ \\ School of Mathematical Sciences, Dalian University of Technology, Dalian, 116024, China \\ (lyc@dlut.edu.cn). \\ Huifu Xu \\ School of Mathematical Sciences, University of Southampton, SO17 1BJ, Southampton, UK \\ (H.Xu@soton.ac.uk) \\ Shu-Jung Sunny Yang \\ Department of Business Administration, College of Management, National Taiwan University, \\ Taipei, 10617, Taiwan (R.O.C.) (sj.sunny.yang@gmail.com) \\ Jin Zhang ${ }^{2}$ \\ Department of Mathematics, Hong Kong Baptist University, Kowloon Tong, Hong Kong, \\ (zhangjin@hkbu.edu.hk)
}

July 16, 2017

\begin{abstract}
We develop several distributionally robust equilibrium models, following the recent research surge of robust game theory, in which some or all of the players in the games lack of complete information on the true probability distribution of underlying uncertainty but they need to make a decision prior to the realization of such uncertainty. We start with a distributionally robust Nash equilibrium model where each player uses partial information to construct a set of distributions and chooses an optimal decision on the basis of the worst distribution rather than the worst scenario to hedge the risk arising from ambiguity of the true probability distribution. We investigate the existence of equilibrium, develop a numerical scheme for its computation, and consider special cases where the distributionally robust Nash equilibrium model can be reformulated as an ordinary deterministic Nash equilibrium. We then extend our modeling scheme to two possible frameworks of distributionally robust Stackelberg setting: a distributionally robust follower model and a distributionally robust leader model. These two frameworks are employed to study an innovative problem of hierarchical competition in a supply chain where a buyer not only invests in its own capacity to supply an end-product market

\footnotetext{
${ }^{1}$ The work of this author was carried out in the School of Mathematical Sciences, University of Southampton. His work is supported in part by EPSRC grant EP/M003191/1 and NSFC Grant \#11571056.

${ }^{2}$ This author was supported by the NSFC NSFC (No. 11401300, 11601458), HKBU FRG1/16-17/007, RCNACAN-ZHANG J.
} 
under demand uncertainty but also outsources a certain amount of market supplies to multiply competing suppliers who invest in capacity for obtaining the buyer's orders. In this application, we show that the buyer has more incentives to invest in capacity whereas the suppliers have less to do so when those suppliers are confronted with more demand uncertainty in the end-product market over the buyer.

Key words. Game theory, distributionally robust Nash equilibrium, distributionally robust Stackelberg models, risk-averse equilibrium models, hierarchical capacity investment

\section{Introduction}

Nash equilibrium has been an important modeling paradigm for optimal decision making problems which involve several decision makers who are in a competitive relationship. When the underlying data of these decision making problems contain uncertain parameters, a stochastic Nash equilibrium model is a must need. Over the past few decades, various stochastic Nash equilibrium models have been proposed depending on the decision-making processes. To be more specific, consider a stochastic Nash equilibrium problem where players need to make decisions prior to the realization of uncertainty and they do so by minimizing their expected disutility, that is, each player $i, i=1, \cdots, m$, sets out its optimal decision by solving an optimization problem

$$
\min _{y_{i} \in Y_{i}} \mathbb{E}_{P}\left[f_{i}\left(y_{i}, y_{-i}, \xi\right)\right],
$$

where $Y_{i} \subset \mathbb{R}^{n_{i}}$ denotes the set of feasible decisions by player $i, y_{-i}$ stands for the decision vectors of its rivals, $\xi$ is a $k$-dimensional random vector defined on probability space $(\Xi, \mathcal{F}, P)$ with support set $\Xi$ representing the underlying uncertainty (e.g., market demand, supply reliability), $f_{i}(\cdot)$ is real-valued continuous function defined on $\mathbb{R}^{n} \times \mathbb{R}^{k}$ with $n=\sum_{i=1}^{m} n_{i}$, and the mathematical expectation $\mathbb{E}[\cdot]$ is taken with respect to the probability distribution of $\xi$.

Stochastic Nash equilibrium models described as the above are ubiquitous in operations literature [10]. For instance, if manufacturing firms compete simultaneously on capacity investment and production, Haurie et al. [24] show that each firm chooses its optimal decision by solving a two-stage stochastic optimization problem. If firms compete sequentially on capacity investment and production (i.e., capacity investment before the realization of demand uncertainty and production after the realization of demand uncertainty), this capacity and production problem becomes a two-stage stochastic equilibrium programs with equilibrium constraints (SEPEC); the relevant discussions can be found in DeMiguel and $\mathrm{Xu}$ [14] for a two-stage multiple-leader stochastic Stackelberg Nash-Cournot model and Henrion and Römisch [25] for the two-stage SEPEC models to study competition in electricity markets. 
We argue that a stochastic Nash equilibrium problem is far trickier than the above discussion when the decision maker does not have complete information on the distributions of random variables; for example, some firms have less information on the distribution of market demand than the other firms in the aforementioned capacity investment and production problems. The operations literature suggests a reasonable option to choose an optimal decision on the basis of extreme values of random variables in order to mitigate the risks while the decision maker does not have any information other than the range of random variables. This sort of decision making framework is known as robust optimization. In the past two decades, robust optimization has been well studied in theories, algorithms and applications, see the monograph by Ben-tal et al. [6] and the survey by Bertsimas et al. [7] for the recent development on robust optimization. In particular, Aghassi and Bertsimas [1] make the first attempt to investigate robust games. They consider a distribution-free model of incomplete-information finite games, both with and without private information, in which the players use a robust optimization approach to contend with payoff uncertainty, see also $[21,28]$ and references therein.

The above discussion motivates us to consider an alternative and possibly less conservative Nash equilibrium concept by following the idea of distributionally robust optimization (DRO for short) to consider that the players in a game are lacking in complete information on the true probability distribution of underlying uncertainty but they can use available information such as historical data, sample information, or subjective judgments to construct a set of distributions which contains or approximates the true distribution. The optimal decision is consequently chosen on the basis of the worst distribution to hedge against the risk arising from ambiguity of the true probability distribution. Over the past decade, DRO approach has been intensively studied for a range of stochastic programming models from one stage to multistage and chanceconstrained problems, see $[16,8,48,20,22,23,27,29,47,49]$ and references therein. In contrast, research on distributionally robust games and equilibrium problems is still in its infancy with a few papers available to date. Qu and Goh [36] seem to be the first to consider a distributionally robust formulation of a stochastic finite game where each player uses the worst probability distribution rather than the worst scenario as in [1] to tackle incomplete information of extraneous uncertainty. Sun and Xu [45] present a stability analysis of distributionally robust Nash equilibrium when players gain more and more information. Ahipasaoglu et al. [2] study the DRO stochastic user equilibrium where the players only have the information of the first and second moments of the random variables. Singh et al. [41] consider robust finite chance-constrained games and study the existence of mixed-strategy Nash equilibrium. Along that direction, Loizou $[31,32]$ proposes a distributionally robust Nash equilibirum model with each player's objective being Conditional Value at Risk (CVaR for short). In the case where the threshold of CVaR is zero, his model coincides with $\mathrm{Qu}$ and Goh's model. One of the main focuses of Loizou's work is 
to investigate cases where distributionally robust games are equivalent to Nash games without private information, that is, to reformulate the distributionally robust games as deterministic Nash games when the ambiguity sets have some special structures.

The existing research on distributionally robust games in the literature focuses on finite games where each player plays a mixed (pure) strategy over finite action space, it is unclear how the established results can be applied to continuous Nash games particularly in terms of numerical methods for computing an equilibrium. Indeed, the numerical method for distributionally robust finite Nash games is still in infancy, with the main streamline being based on the dual reformulation when the ambiguity set has some specific structure, see [36, 31] for details. This paper aims to fill out the gap. We start by investigating the existence of the equilibrium for distributionally robust Nash (DRNE for short) model, and algorithms for computing it, and then followed by analyzing distributionally robust Stackelberg problems. Since the dual formulation of DRNE can be atypically sophisticated, especially the Stackelberg model, the prevailing dual formulation technique in the literature for DRO problem is no longer a powerful tool here. Instead, we focus on some special cases where either the DRNE can be significantly simplified or they are related to risk averse Nash equilibrium models.

We consider a real-world case related to distributionally robust Stackelberg models. As a well-known fact documented in the operations and supply chain literature, the sector of consumer electronics is subject to tight capacity constraints. When launching new products, quantity produced is limited by existing workforce, facilities, and/or raw material availability. The production capacity is often reached and limits the possible output quantity for seizing further market opportunities. Therefore, outsourcing is a key strategy that firms use to overcome capacity limit when launching new products. By outsourcing, firms (henceforth buyers, to distinguish them from suppliers) can produce the amount of products above the installed capacity in house, while contracting out certain amount of production to suppliers who may need to expand their capacity, as well, for obtaining the buyers' orders. While outsourcing reduces a buyer's pressure on capacity expansion, it gives rise to the phenomenon whereby the suppliers can directly compete with the buyer by supplying the residual capacity to the product market. Specifically, outsourcing opens a window of opportunity for the suppliers to enter the product market. One prominent example of this hierarchical capacity competition across a supply chain is HTC who began as a contract cell phone manufacturer and later entered the smartphone market with its own brand [52]. To address this hierarchical capacity competition problem in a supply chain, we therefore propose two distributionally robust Stackelberg models, distributionally robust follower model and distributionally robust leader model. The first model suits the case that the leader (i.e., the buyer in our case) dominates the market with complete information on market uncertainty, however, the followers (i.e., the suppliers in our case) only have partial information. 
The second model is suitable to the case where reversely the followers have complete information on the market uncertainty.

To sum up, the main contributions of our work are summarized as follows.

- We propose a distributionally robust formulation for stochastic continuous Nash equilibrium problems, which extends the recent work by Aghassi and Bertsimas [1], Qu and Goh [36] and Loizou [31, 32]. We investigate the existence of equilibrium and develop a numerical scheme for computing it. Special cases are considered where the distributionally robust Nash equilibrium model can be reformulated as ordinary deterministic Nash equilibrium.

- We propose two distributionally robust formulations for stochastic Stackelberg games: a distributionally robust follower model and a distributionally robust leader model. We demonstrate that the former can be recast into a mathematical program with equilibrium constraints (MPEC for short) model whereas the latter can be reformulated as a standard distributionally robust optimization problem under some specific circumstances.

- We use the proposed distributionally robust follower model to analyze a hierarchical capacity competition problem and conduct a numerical study. The results underscore the importance of informational completeness on uncertainty and some managerial insights are derived.

The rest of the paper is organized as follows. In section 2, we give an exact definition of the DRNE model and investigate existence of an equilibrium. An algorithmic framework is proposed for solving the DRNE problem. In section 3, we discuss two special cases where the ambiguity sets are defined through prior moments and linked to coherent risk measure. The former allows us to reformulate the DRNE model into an ordinary deterministic Nash equilibrium model while the latter relates the DRNE to standard risk-averse Nash games. In section 4, we move on to discuss distributionally robust Stackelberg model from two perspectives, a distributionally robust follower model and a distributionally robust leader model. Finally, in section 5 we apply the proposed models to hierarchical capacity competition in a supply chain where a buyer not only invests in its own capacity to supply an uncertain market but also outsources a certain amount of market supply to multiple competing suppliers who invest in capacity for obtaining the buyer's orders.

\section{Distributionally robust Nash equilibrium}

Stochastic Nash equilibrium (SNE for short) is an important solution concept in game theory and has found many applications in engineering, economics, operations, and marketing, see [51, 
37, 39] and the references therein. An important assumption in the stochastic Nash equilibrium models is that the true probability distribution of underlying uncertainty is known. However, in many practical circumstances, we may only have partial information such as the range of uncertain parameters, historical data, or subjective judgment of the probability distributions. Assuming each player is risk averse against ambiguity of the true probability distribution, then the resulting Nash equilibrium may be different from stochastic equilibrium. This motivates us to investigate the former in terms of the models and numerical methods.

To start with, an individual player $i$ 's decision-making problem can be formulated as:

$$
\min _{y_{i} \in Y_{i}} \max _{P \in \mathcal{P}_{i}} \mathbb{E}_{P}\left[f_{i}\left(y_{i}, y_{-i}, \xi\right)\right]
$$

where $f_{i}(\cdot)$ is a continuous function from $\mathbb{R}^{n} \times \mathbb{R}^{k}$ to $\mathbb{R}, n=\sum_{i=1}^{m} n_{i}, y_{i} \in Y_{i} \subset \mathbb{R}^{n_{i}}$ denotes player $i$ 's decision vector, and $y_{-i}$ represents the decision variables of its rivals. A key element in this formulation is the maximum operation w.r.t. $P$ which means the player bases its optimal decision on the worst expected value of $f_{i}$ from the set of distributions $\mathcal{P}_{i}$ which is constructed from available information. Assuming each player takes a decision based on (2.1), then we look into a situation where no player can get better off by unilaterally changing its position.

Definition 2.1 An $m$-tuple $\left(y_{1}^{*}, \cdots, y_{m}^{*}\right)$ is called an equilibrium of distributionally robust Nash equilibrium (DRNE) if

$$
y_{i}^{*} \in \arg \min _{y_{i} \in Y_{i}} \max _{P \in \mathcal{P}_{i}} \mathbb{E}_{P}\left[f_{i}\left(y_{i}, y_{-i}^{*}, \xi\right)\right], \text { for } i=1, \cdots, m \text {. }
$$

This type of model is first studied by $\mathrm{Qu}$ and Goh [36] for a two-players finite game. In the case where $\mathcal{P}_{i}, i=1, \cdots, m$, contains a singe distribution, (2.2) collapses to stochastic Nash equilibrium problem. On the other hand, when $\mathcal{P}_{i}$ comprises the set of all the probability measures (over the support of $\xi$ induced by $\xi$ ), (2.2) reduces to the distribution-free robust model proposed by Aghassi and Bertsimas [1]. Note that the objective of each player in this model is the expected value of a random function. It is possible to consider the case where the objective is a risk measure such as CVaR in $[31,32]$ and more broadly distortion risk measure which would cover any law invariant coherent risk measure, see [33, Section 4].

In what follows, we investigate the existence of equilibrium of the DRNE model and numerical schemes for computing such an equilibrium.

Assumption 2.1 Let $f_{i}(\cdot)$ be defined as in (2.2). The following assertions hold for $i=1, \cdots, m$.

(a) $f_{i}(\cdot)$ is a continuous function and for each fixed $\left(y_{-i}, \xi\right), f_{i}\left(\cdot, y_{-i}, \xi\right)$ is convex over $Y_{i}$. 
(b) $Y:=Y_{1} \times \cdots \times Y_{m}$ is a compact set.

(c) $\mathbb{E}_{P}\left[f_{i}\left(y_{i}, y_{-i}, \xi\right)\right]$ is finite valued for any $y \in Y$ and $P \in \mathcal{P}_{i}$.

(d) $\mathcal{P}_{i}$ is weakly compact. ${ }^{3}$

Assumption 2.1 ensures that each player's objective function is finite valued and it is convex w.r.t. its decision vector. Indeed, under Assumption 2.1 (a)-(b), $\mathbb{E}_{P}\left[f_{i}\left(y_{i}, y_{-i}, \xi\right)\right], i=1, \cdots, m$, is well defined and it is convex w.r.t. $y_{i}$ for each fixed $y_{-i}$. Conditions (c)-(d) ensure that optimum of the inner maximization problem can be achieved by some probabilities $P \in \mathcal{P}_{i}$. Under Assumption 2.1, we may reformulate the problem (2.2) as a single optimization problem.

Proposition 2.1 Let

$$
\phi(x, y):=\sum_{i=1}^{m} \max _{P \in \mathcal{P}_{i}} \mathbb{E}_{P}\left[f_{i}\left(x_{i}, y_{-i}, \xi\right)\right] .
$$

Under Assumption 2.1, $y^{*}$ is an equilibrium of DRNE (2.2) if and only if

$$
y^{*} \in \arg \min _{x \in Y} \phi\left(x, y^{*}\right)
$$

Proof. The reformulation is well known for deterministic Nash equilibrium, see for example Rosen [40]. Here we provide a proof as the DRNE involves minimax operations. The "if" part follows from the fact that if $y^{*}$ is not an equilibrium, then there exists some $1 \leq i_{0} \leq m$ and $\bar{x}_{i_{0}} \in Y_{i_{0}}$ such that

$$
\max _{P \in \mathcal{P}_{i_{0}}} \mathbb{E}_{P}\left[f_{i_{0}}\left(\bar{x}_{i_{0}}, y_{-i_{0}}^{*}, \xi\right)\right]<\max _{P \in \mathcal{P}_{i_{0}}} \mathbb{E}_{P}\left[f_{i_{0}}\left(y_{i_{0}}^{*}, y_{-i_{0}}^{*}, \xi\right)\right]
$$

Let $\bar{x}:=\left(y_{1}^{*}, \cdots, y_{i_{0}-1}^{*}, \bar{x}_{i_{0}}, y_{i_{0}+1}^{*}, \cdots, y_{m}^{*}\right)$, we have $\phi\left(\bar{x}, y^{*}\right)<\phi\left(y^{*}, y^{*}\right)$, a contradiction. The "only if" part is obvious as

$$
\max _{P \in \mathcal{P}_{i}} \mathbb{E}_{P}\left[f_{i}\left(x_{i}, y_{-i}^{*}, \xi\right)\right] \geq \max _{P \in \mathcal{P}_{i}} \mathbb{E}_{P}\left[f_{i}\left(y_{i}^{*}, y_{-i}^{*}, \xi\right)\right]
$$

for $i=1, \cdots, m$ and summing up w.r.t. $i$ on both sides of the inequality shows $y^{*}$ is a global minimizer.

Based on Proposition 2.1, we have the following existence results for DRNE (2.2).

\footnotetext{
${ }^{3} \mathrm{~A}$ set of probability measures $\mathcal{A}$ is said to be weakly compact if every sequence $\left\{P_{N}\right\} \subset \mathcal{A}$ contains a subsequence $\left\{P_{N^{\prime}}\right\}$ and $P \in \mathcal{A}$ such that $P_{N^{\prime}}$ converges to $P$ weakly.
} 
Theorem 2.1 Under Assumption 2.1, DRNE (2.2) has an equilibrium.

Proof. Under Assumption 2.1, $\mathbb{E}_{P}\left[f_{i}\left(\cdot, y_{-i}, \xi\right)\right]$ is continuous and convex for every $P \in \mathcal{P}_{i}$ and $i=1, \cdots, m$. The supremum preserves the convexity and, under weakly compactness of $\mathcal{P}_{i}$, the continuity. Therefore $\phi(\cdot, y)$ is continuous and convex w.r.t. $x$ on $Y$ for any fixed $y \in Y$.

The existence of an optimal solution to $\min _{x \in Y} \phi(x, y)$ follows from compactness of $Y$ under Assumption 2.1 (c). To complete the proof, we are left to show the existence of $y^{*} \in Y$ such that

$$
y^{*} \in \arg \min _{x \in Y} \phi\left(x, y^{*}\right)
$$

Let $\Gamma(y)$ be the set of optimal solutions to $\min _{x \in Y} \phi(x, y)$ for each fixed $y \in Y$. Then $\Gamma(y) \subset Y$. By the convexity of $\phi(\cdot, y), \Gamma(y)$ is a convex set. Moreover, it is easy to show that $\Gamma(y)$ is closed, that is, for $y_{k} \rightarrow \bar{y}$ and $x_{k} \in \Gamma\left(y_{k}\right)$ with $x_{k} \rightarrow \bar{x}, \bar{x} \in \Gamma(\bar{y})$. Further, it follows by [4, Theorem 4.2.1] that $\Gamma(\cdot)$ is upper semi-continuous on $Y$. By Kakutani's fixed point theorem [26], there exists $y^{*} \in Y$ such that $y^{*} \in \Gamma\left(y^{*}\right)$.

The next key step towards understanding the DRNE model and applying it to practical problems is to develop an efficient numerical method for identifying an equilibrium of DRNE (2.2). Obviously, the DRNE model is mathematically more sophisticated than SNE and therefore requires new methods different from the existing numerical schemes.

If the objective functions are convex w.r.t. each player's decision vector, we may consider an iterative scheme where at each iteration we solve a single minimax optimization problem. 


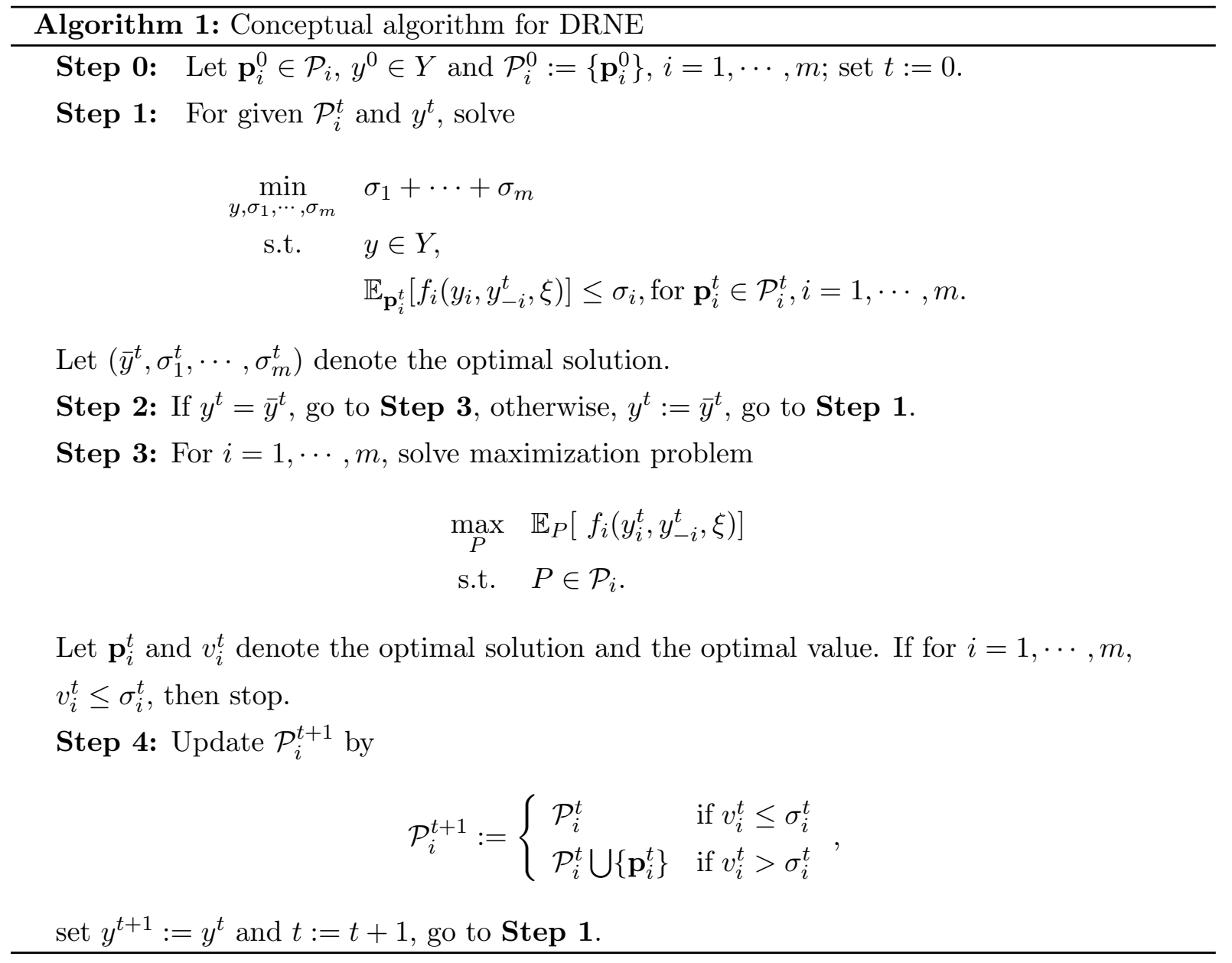

Algorithm 1 is motivated by a similar algorithm proposed by $\mathrm{Xu}$, Liu and Sun [50] for solving a distributionally robust portfolio problem. Steps 1-2 are designed to solve

$$
\min _{x \in Y} \phi^{t}\left(x, y^{t}\right):=\sum_{i=1}^{m} \max _{P \in \mathcal{P}_{i}^{t}} \mathbb{E}_{P}\left[f_{i}\left(x_{i}, y_{-i}^{t}, \xi\right)\right],
$$

or equivalently to find an equilibrium of

$$
\min _{y_{i} \in Y_{i}} \max _{P \in \mathcal{P}_{i}^{t}} \mathbb{E}_{P}\left[f_{i}\left(y_{i}, y_{-i}, \xi\right)\right], \text { for } i=1, \cdots, m .
$$

At the end of the Step 2, we obtain $\bar{y}^{t}$ which solves (2.4). Note that the objective function of (2.3) is separable, therefore $\sigma_{i}^{t}$ corresponds to the optimal value of (2.4).

Step 3 is to examine whether $\bar{y}^{t}$ is an equilibrium of DRNE (2.2). Observe that $v_{i}^{t} \geq \sigma_{i}^{t}$ because $\mathcal{P}_{i}^{t} \subset \mathcal{P}_{i}$. In the case where the equality holds for all $i=1, \cdots, m$, we can replace $\max _{P \in \mathcal{P}_{i}^{t}} \mathbb{E}_{P}\left[f_{i}\left(y_{i}, y_{-i}, \xi\right)\right]$ with $\max _{P \in \mathcal{P}_{i}} \mathbb{E}_{P}\left[f_{i}\left(y_{i}, y_{-i}, \xi\right)\right]$ for $y=y^{t}$ and this means $\bar{y}^{t}$ solves DRNE (2.2).

From the discussions above, we conclude that if the algorithm terminates in a finite number of iterations $t$, then $y^{t}$ (or $\bar{y}^{t}$ ) must be an equilibrium to DRNE (2.2). The theorem below 
addresses the case that Algorithm 1 does not stop in a finite number of iterations.

Theorem 2.2 Let $\left\{y^{t}\right\}$ be generated by Algorithm 1. Assume that the conditions of Theorem 2.1 are satisfied. Then every accumulation point ${ }^{4}$ of sequence $\left\{y^{t}\right\}$ is an equilibrium of DRNE (2.2).

Proof. Let $y^{*}$ be an accumulation point of sequence $\left\{y^{t}\right\}$. By the procedures of Algorithm 1 , for each $i=1, \cdots, m$, the sequence $\left\{\mathcal{P}_{i}^{t}\right\}$ is monotonically increasing in the sense that $\mathcal{P}_{i}^{t} \subset \mathcal{P}_{i}^{t+1}$. As $\mathcal{P}_{i}, i=1, \cdots, m$, is a compact set, there exists $\mathcal{P}_{i}^{*} \subset \mathcal{P}_{i}$ such that

$$
\lim _{t \rightarrow \infty} \mathcal{P}_{i}^{t}=\mathcal{P}_{i}^{*}
$$

Taking advantage of the analysis on case that Algorithm 1 stops at finite steps, it is sufficient to show that

$$
\max _{P \in \mathcal{P}_{i}} \mathbb{E}_{P}\left[f_{i}\left(y_{i}^{*}, y_{-i}^{*}, \xi\right)\right]=\max _{P \in \mathcal{P}_{i}^{*}} \mathbb{E}_{P}\left[f_{i}\left(y_{i}^{*}, y_{-i}^{*}, \xi\right)\right], \quad i=1, \cdots, m
$$

Since for each $t$ and $i=1, \cdots, m$,

$$
\max _{P \in \mathcal{P}_{i}} \mathbb{E}_{P}\left[f_{i}\left(y_{i}^{t}, y_{-i}^{t}, \xi\right)\right]=\max _{P \in \mathcal{P}_{i}^{t+1}} \mathbb{E}_{P}\left[f_{i}\left(y_{i}^{t}, y_{-i}^{t}, \xi\right)\right]
$$

Then, we have

$$
\begin{aligned}
\max _{P \in \mathcal{P}_{i}} \mathbb{E}_{P}\left[f_{i}\left(y_{i}^{*}, y_{-i}^{*}, \xi\right)\right] & =\lim _{t \rightarrow \infty} \max _{P \in \mathcal{P}_{i}} \mathbb{E}_{P}\left[f_{i}\left(y_{i}^{t}, y_{-i}^{t}, \xi\right)\right] \\
& =\lim _{t \rightarrow \infty} \max _{P \in \mathcal{P}_{i}^{t+1}} \mathbb{E}_{P}\left[f_{i}\left(y_{i}^{t}, y_{-i}^{t}, \xi\right)\right] \\
& =\lim _{t \rightarrow \infty} \max _{P \in \mathcal{P}_{i}^{t}} \mathbb{E}_{P}\left[f_{i}\left(y_{i}^{t}, y_{-i}^{t}, \xi\right)\right] \\
& =\max _{P \in \mathcal{P}_{i}^{*}} \mathbb{E}_{P}\left[f_{i}\left(y_{i}^{*}, y_{-i}^{*}, \xi\right)\right]
\end{aligned}
$$

where the first equality follows from [9, Proposition 4.4] and the second equality follows (2.5). The proof is complete.

It might be helpful to note that Algorithm 1 only provides a conceptual numerical framework for solving DNRE (2.2) under the abstract form of the ambiguity sets $\mathcal{P}_{i}, i=1, \cdots, m$. For instance, Step 3 requires to solve an optimization problem w.r.t. probability measure which is difficult if $\mathcal{P}_{i}$ consists of continuous distributions. In that case, further discretization might be needed, see $\mathrm{Xu}$ et al. [50] where a discretion scheme is proposed for the case when the ambiguity set is defined by generalized moment conditions. Note also that when $\mathcal{P}_{i}$ has a specific structure, it might be possible to reformulate each player's optimization problem as

\footnotetext{
${ }^{4}$ We say $c^{*}$ is an accumulation point of sequence $\left\{c^{n}\right\}$ if there is a subsequence $\left\{c^{k}\right\}$ of $\left\{c^{n}\right\}$ converges to $c^{*}$.
} 
a semidefinite programming problem (SDP for short) and then as a system of Karush-KuhnTucker condition, see Qu and Goh [36] and Loizou [31, 32] for distributionally robust finite Nash games.

At the end of this section, we provide a simple example to explain the difference between stochastic Nash equilibrium and distributionally robust Nash equilibrium.

Example 2.1 (Boxed pigs) A pig and a piglet are put in a box with a lever at one end of the box and dispenses food at the other end. So the pig that presses the lever must run to the other end to eat, and by the time it gets there, the other pig has eaten most, but not all the food. The big pig is dominant and the piglet is subordinate. Therefore big pig is able to prevent piglet from getting any food when both are at the food. When a pig presses the lever, it will incur a disutility of $\alpha$ units (which can be interpreted as the energy to be consumed), and $\xi$ units (a random variable taking integer values) of food will be released at the dispenser.

We assume that the pigs can reason like game theorists and they need to decide as to whether to press the lever or wait at the dispenser. Since the big pig is a 'dominant' player, if it gets the dispenser first (wait at the dispenser) or at the same time (both press the lever and then run to the dispenser) with the small pig, it will get the following amount of the food

$$
p_{d}(\xi):= \begin{cases}\xi, & \xi \leq 9, \\ 9+\ln (\xi-9), & \xi \geq 10\end{cases}
$$

whereby the small pig gets the rest. If, instead, the small pig waits at the dispenser first, it will get

$$
p_{s}(\xi):= \begin{cases}\xi, & \xi \leq 4, \\ 4+\ln (\xi-4), & \xi \geq 5,\end{cases}
$$

and the big pig gets the rest. Let $\alpha=6$ for the big pig and $\alpha=2$ for the small pig. Table 2.1

\begin{tabular}{|c|c|c|c|}
\hline & & Small $\mathrm{p}$ & \\
\hline & & $\mathrm{P}$ & $\mathrm{W}$ \\
\hline & $\mathrm{P}$ & $\left(p_{d}(\xi)-6, \xi-p_{d}(\xi)-2\right)$ & $\left(\xi-p_{s}(\xi)-6, p_{s}(\xi)\right)$ \\
\hline 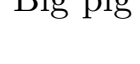 & $\mathrm{W}$ & $\left(p_{d}(\xi), \xi-p_{d}(\xi)-2\right)$ & $(0,0)$ \\
\hline
\end{tabular}
summarizes the payoffs for the strategies Press (P) the panel and Wait (W) at the dispenser.

Table 1: Boxed Pigs

Suppose that the random variable $\xi$ may follow the two potential distributions $P_{1}(\xi=4)=\frac{1}{4}$ and $P_{1}(\xi=15)=\frac{3}{4}$ or $P_{2}(\xi=4)=\frac{3}{4}$ and $P_{2}(\xi=15)=\frac{1}{4}$.

In what follows, we compare the equilibria when the pigs play a stochastic equilibrium and robust equilibrium. In Table 2, we display the outcomes of the expected utilities when the pigs 
play a stochastic game with $P_{1}$. It is easy to see that there are two SNE: $(\mathrm{W}, \mathrm{P})$ and $(\mathrm{P}, \mathrm{W})$, which means one of the pigs will press the lever.

Suppose that the two pigs know that the true probability could be $P_{1}$ or $P_{2}$ but there is an ambiguity of which one it is. In that case, they may take a robust strategy by considering the worst probability distribution in its decision making. Table 3 displays four possible outcomes and the DRNE is $(\mathrm{W}, \mathrm{W})$, no one will press the lever.

\begin{tabular}{|c|c|c|c|}
\hline \multirow{4}{*}{ Big pig } & \multicolumn{3}{|c|}{ Small pig } \\
\hline & \multirow[b]{2}{*}{$\mathrm{P}$} & $\mathrm{P}$ & $\mathrm{W}$ \\
\hline & & $(2.0938,1.1562)$ & $(0.4516,5.7984)$ \\
\hline & \multirow[t]{4}{*}{$\mathrm{W}$} & $(8.0938,1.1562)$ & $(0,0)$ \\
\hline & & Table 2: SNE mod & with $P_{1}$ \\
\hline \multirow{4}{*}{ Big pig } & & \multicolumn{2}{|c|}{ Small pig } \\
\hline & & $\mathrm{P}$ & $\mathrm{W}$ \\
\hline & $\mathrm{P}$ & $(-0.302,-0.948)$ & $(-2.8495,3.5995)$ \\
\hline & $\mathrm{W}$ & $(5.698,-0.948)$ & $(\mathbf{0}, \mathbf{0})$ \\
\hline
\end{tabular}

Table 3: DRNE model with $\left\{P_{1}, P_{2}\right\}$

The example shows that the SNE and DRNE models generate complete different equilibrium outcomes. From our perspective (modelers), we may not know which type of game is played out in practice. However, if a player does not have complete information on the true probability distribution, it is reasonable for us to assume that the player would take a robust strategy. By comparing our modelling/analytical equilibrium results with practically equilibrium outcomes, we may provide practitioners with some business insights as to whether an SNE model or a DRNE model is better suited to the practice. In others words, our DRNE model may provide an alternative mathematical modelling paradigm for understanding and/or predicting market competition and giving the regulator some guidance.

To explain how Algorithm 1 works in this example, we highlight the moves following the algorithmic procedures as follows.

(i) Set $\mathcal{P}_{1}^{0}:=\left\{P_{1}\right\}$ and $\mathcal{P}_{2}^{0}:=\left\{P_{1}\right\}$. Return an equilibrium $(W, P)$ (or $(\mathrm{P}, \mathrm{W})$ ) by going through Steps 1-2 (based on calculations displayed in Table 2).

(ii) Check the optimality of $(W, P)$ (or $(\mathrm{P}, \mathrm{W}))$ in Step 3. Both of the two pigs fail.

(iii) Update $\mathcal{P}_{1}^{1}=\left\{P_{1}, P_{2}\right\}$ and $\mathcal{P}_{2}^{1}:=\left\{P_{1}, P_{2}\right\}$. 
(iv) Go to Steps 1-2 and return with an equilibrium $(W, W)$ (based on calculations in the Table 3). Check the optimality in Step 3, and clearly it is satisfied.

\section{Reformulation of DRNE}

The DRNE model and the numerical scheme considered in the preceding section have not taken into account the specific structure of the ambiguity set which reflects player's perception of the distribution of underlying uncertainty. In this section, we investigate some special cases where (a) the ambiguity set is constructed through prior moment information and (b) each player's objective is to minimize the tail risk measure such as entropic risk measure and conditional value at risk (CVaR for short). This will enable us to simplify the mathematical structure of the DRNE model and propose simpler numerical methods to solve it.

\subsection{DRNE with moment information}

In this subsection, we consider the case that the ambiguity set is defined through moment condition of the random variable. The underlying consideration of moment condition is that given some historical data, it is often easier to estimate the moments of random parameters than to derive their probability distributions. Here we focus on two kinds of ambiguity sets considered in the recent works $[13,35]$. By employing the new results in $[13,35]$, we can get an explicit solution to the inner maximization of each player's distributionally robust problem (2.1) and then reformulate the distributionally robust Nash equilibrium as a deterministic Nash game. Indeed, Loizou [31, 32] first studies the equivalence between DRNE and standard Nash equilibrium. He shows that in finite action games, when each player's objective is a linear function of the underlying random variables, the DRNE is equivalent to the standard Nash equilibrium when the first moment of the random variables is known. It is not difficult to extend Loizou's results to continuous DRNE under the same setting and therefore our focus is on the nonlinear case.

First, consider the case that

$$
\mathcal{P}:=\left\{P \in \mathscr{P}(\Xi): P(\xi \in \Xi)=1, \quad \mathbb{E}_{P}[\xi]=\mu, \quad \mathbb{E}_{P}[\psi(\xi)] \leq 0\right\},
$$

where $\mathscr{P}(\Xi)$ denotes the set of all probability measures (over the support $\Xi$ ), $\mu$ is the mean value of random variable $\xi$, and $\psi(\cdot)$ is a convex functions. (3.6) is first considered by Delage et al. [13] and they study a two-stage mixed-integer stochastic linear programs. Ambiguity set (3.6) can capture well the information we would have in hand in early steps of uncertainty 
assessment. The following special case is first considered in [12]:

$$
\mathcal{P}:=\left\{P \in \mathscr{P}(\Xi): \quad \begin{array}{ll}
\mathbb{E}_{P}[\xi-\mu]^{T} \Sigma^{-1} \mathbb{E}_{P}[\xi-\mu] \leq \gamma_{1} \\
& 0 \preceq \mathbb{E}_{P}\left[(\xi-\mu)(\xi-\mu)^{T}\right] \preceq \gamma_{2} \Sigma
\end{array}\right\},
$$

where $\gamma_{1}$ and $\gamma_{2}$ are nonnegative constants and $\Sigma$ is the covariance of $\xi$. Based on the results in [13], we have the following explicit expression of each player's objective.

Example 3.1 Consider the case where $f_{i}\left(y_{i}, y_{-i}, \cdot\right)$ is concave with respect to $\xi$ for each fixed $y$ and $\mathcal{P}_{i}$ is defined in the form of (3.6). It follows by [13, Proposition 1]

$$
\max _{P \in \mathcal{P}_{i}} \mathbb{E}_{P}\left[f_{i}\left(y_{i}, y_{-i}, \xi\right)\right]=f_{i}\left(y_{i}, y_{-i}, \mu\right),
$$

where $\mu$ is the mean value of random variable $\xi$. Consequently, we may reformulate the DRNE $(2.2)$ as:

$$
y_{i}^{*} \in \arg \min _{y_{i} \in Y_{i}} f_{i}\left(y_{i}, y_{-i}^{*}, \mu\right), \text { for } i=1, \cdots, m .
$$

The latter is a deterministic Nash equilibrium problem so that well-established numerical methods in the literature are ready to be used.

Another case allowing us to develop a simple reformulation as in Example 3.1 is when the ambiguity set is defined as:

$$
\mathcal{P}:=\left\{P \in \mathscr{P}(\Xi): \mathbb{E}_{P}[\xi]=\mu, \quad \mathbb{E}_{P}\left[\left|\xi_{i}-\mu_{i}\right|\right]=d_{i}, \quad \forall i, \xi_{i} \perp \xi_{j}, \forall i \neq j\right\},
$$

where $\Xi:=\left[a_{1}, b_{1}\right] \times \cdots \times\left[a_{k}, b_{k}\right]$ and $\xi_{i} \perp \xi_{j}$ denotes the stochastic independence of components $\xi_{i}$ and $\xi_{j}$. Postek et al. [35] derive exact robust counterparts of expected feasibility of convex constraints by employing some more earlier results [5] when the ambiguity set is defined by (3.7). We use their results to provide the closed form of DRNE model.

Example 3.2 Suppose that (a) for any given $y \in Y, f_{i}\left(y_{i}, y_{-i}, \cdot\right)$ is concave with respect to $\xi$, (b) $\mathcal{P}_{i}$ is defined in the form of (3.7). Then by [35, Section 2.3]

$$
\max _{P \in \mathcal{P}_{i}} \mathbb{E}_{P}\left[f_{i}\left(y_{i}, y_{-i}, \xi\right)\right]=\tilde{f}_{i}\left(y_{i}, y_{-i}\right):=\sum_{\kappa \in\{1,2,3\}^{k}} \Pi_{i=1}^{k} p_{\kappa_{i}}^{i} f\left(y_{i}, y_{-i}, \lambda_{\kappa_{1}}^{1}, \cdots, \lambda_{\kappa_{k}}^{k}\right),
$$

where $\{1,2,3\}^{k}$ denotes the set of all enumerating over $3^{k}$ permutations of outcomes $a_{i}, \mu_{i}, b_{i}$ of $\xi_{i}$,

$$
\lambda_{1}^{i}=a_{i}, \quad \lambda_{2}^{i}=\mu_{i}, \quad \lambda_{3}^{i}=b_{i}, \quad \text { for } i=1, \cdots, k
$$

and

$$
p_{1}^{i}=\frac{d_{i}}{2\left(\mu_{i}-a_{i}\right)}, \quad p_{2}^{i}=1-\frac{d_{i}}{2\left(\mu_{i}-a_{i}\right)}-\frac{d_{i}}{2\left(b_{i}-\mu_{i}\right)}, \quad p_{3}^{i}=\frac{d_{i}}{2\left(b_{i}-\mu_{i}\right)} .
$$


Subsequently, we may reformulate (2.2) as:

$$
y_{i}^{*} \in \arg \min _{y_{i} \in Y_{i}} \tilde{f}_{i}\left(y_{i}, y_{-i}^{*}\right), \text { for } i=1, \cdots, m .
$$

Again, (3.9) is a standard Nash equilibrium.

In the two examples, the specific structure of the prior moment conditions enables us to reformulate the DRNE as a deterministic Nash equilibrium problem. When the moment conditions are presented in a more general form, we might not be able to obtain a closed form for the inner maximization problem. For instance, Wiesemann et al. [48] recently consider a type of ambiguity sets:

$$
\mathcal{P}:=\left\{P \in \mathscr{P}\left(\mathbb{R}^{m_{1}} \times \mathbb{R}^{m_{2}}\right): \mathbb{E}_{P}[\mathbf{A} \xi+\mathbf{B} \tilde{\xi}]=\mathbf{b}, P\left\{(\xi, \tilde{\xi}) \in \mathbf{\Xi}_{\mathbf{i}}\right\} \in\left[\underline{\mathbf{p}_{i}}, \overline{\mathbf{p}_{i}}\right], i \in \mathbf{I}\right\}
$$

where $P$ represents a joint probability distribution of the random vector $\xi \in \mathbb{R}^{m_{1}}$ and some auxiliary random vectors $\tilde{\xi} \in \mathbb{R}^{m_{2}}, \mathbf{A} \in \mathbb{R}^{k \times m_{1}}, \mathbf{B} \in \mathbb{R}^{k \times m_{2}}, \mathbf{I}=\{1, \cdots, I\}$

$$
\boldsymbol{\Xi}_{\mathbf{i}}=\left\{(\xi, \tilde{\xi}): \mathbf{C}_{i} \xi+\mathbf{D}_{i} \tilde{\xi} \preceq \mathcal{K}_{i} \mathbf{c}_{i}\right\}
$$

with $\mathbf{C}_{i} \in \mathbb{R}^{l_{i} \times m_{1}}, \mathbf{D}_{i} \in \mathbb{R}^{l_{i} \times m_{1}}, \mathbf{c}_{i} \in \mathbb{R}^{l_{i}}, \mathcal{K}_{i}$ being proper cone and $y^{\prime \prime} \preceq \mathcal{K}_{i} y^{\prime}$ meaning $y^{\prime}-y^{\prime \prime} \in \mathcal{K}_{i}$. Through dual reformulation, they convert the resulting distributionally robust optimization problem into a tractable semidefinite programming problem. Accordingly, we can easily plug their results [48] into our DRNE model.

Suppose that (a) the confidence set $\boldsymbol{\Xi}_{I}$ is bounded and has probability one, that is, $\mathbf{p}_{I}=$ $\overline{\mathbf{p}_{I}}=1$; (b) the Slater condition holds; (c) the objective function $f_{j}(y, \xi)$ can be written as

$$
f_{j}(y, \xi)=\max _{1 \leq l \leq L} g_{l}(x, \xi)
$$

with $g_{l}(y, \xi)=s_{l}(\xi)^{T} y+t_{l}(\xi), s_{l}(\xi)=\mathbf{S}_{l} \xi+\mathbf{s}_{l}, \mathbf{S}_{l} \in \mathbb{R}^{n \times m_{1}}, \mathbf{s}_{l} \in \mathbb{R}^{n}, t_{l}(\xi)=\mathbf{T}_{l}^{T} \xi+\mathbf{t}_{l}, \mathbf{T}_{l} \in \mathbb{R}^{m_{1}}$ and $\mathbf{t}_{l} \in \mathbb{R}$; (d) for all $i, i^{\prime} \in \mathbf{I}$, either $\boldsymbol{\Xi}_{i} \subset \boldsymbol{\Xi}_{i^{\prime}}, \boldsymbol{\Xi}_{i^{\prime}} \subset \boldsymbol{\Xi}_{i}$ or $\boldsymbol{\Xi}_{i} \cap \boldsymbol{\Xi}_{i^{\prime}}=\emptyset$. By [48, Theorem 1], player $j$ 's distributionally robust optimization problem can be reformulated as

$$
\left.\begin{array}{ll}
\min _{y_{i}, \beta, \kappa, \lambda, \phi} & \mathbf{b}^{T} \beta+\sum_{i \in \mathbf{I}}\left[\overline{\mathbf{p}_{i}} \kappa_{i}-\underline{\mathbf{p}_{i}} \lambda_{i}\right] \\
& \mathbf{c}_{i}^{T} \phi_{i l}+\mathbf{s}_{l}^{T}\left(y_{i}, y_{-i}\right)+\mathbf{t}_{l} \leq \sum_{i^{\prime} \in \mathcal{A}(i)}\left[k_{i^{\prime}}-\lambda_{i^{\prime}}\right] \\
\text { s.t. } & \mathbf{C}_{i}^{T} \phi_{i l}+\mathbf{A}^{T} \beta=\mathbf{S}_{l}^{T}\left(y_{i}, y_{-i}\right)+\mathbf{T}_{l} \\
& \mathbf{D}_{i}^{T} \phi_{i l}+\mathbf{B}^{T} \beta=0
\end{array}\right\}
$$

where $\beta \in \mathbb{R}^{k}, \kappa, \lambda \in \mathbb{R}_{+}^{I}, \phi_{i l} \in \mathcal{K}_{i}^{*}, i \in \mathbf{I}, 1 \leq l \leq L$ and $\mathcal{A}(i)=i \cup\left\{i^{\prime} \in \mathbf{I}, \boldsymbol{\Xi}_{i} \subseteq \boldsymbol{\Xi}_{i^{\prime}}\right\}$.

If $\boldsymbol{\Xi}_{i}$ is described by linear, conic-quadratic or semidefinite inequalities, then we are guaranteed by [48, Theorem 1] that (3.10) is a linear, conic-quadratic or semidefinite programming 
problem. If we further use the Karush-Kuhn-Tucker condition to reformulate the Nash equilibrium, we may have a system of equalities and inequalities. Note that in problem (3.10), player $j$ 's feasible solution set depends on the other players' decision variables, and thus the resulting reformulation of DRNE is a generalized Nash equilibrium problem rather than a simple Nash equilibrium problem as in the two examples. We refer readers to an excellent survey paper by Facchinei and Kanzow [17] for numerical methods and underlying theory for generalized Nash equilibrium.

\subsection{Linking DRNE to risk-averse equilibrium models}

The DRNE model can also be related to stochastic equilibrium models where the objective of each player is a coherent risk measure. The latter has been investigated by a few papers, for example, Ralph and Smeers [37] develop a so-called stochastic endogenous equilibrium framework for pricing risks of risk assets and Ravat and Shanbhag [39] study risk-averse Nash-Cournot games. Singh et al. [41] study the existence of equilibria of chance-constrained games. Loizou [31] proposes a DRNE model where each player employs a distributionally robust CVaR as their objective. In what follows, we start to establish a link between the DRNE model and two risk-averse equilibrium models.

Let $Z$ denote the loss of an investment which is a random variable defined over space $\mathbb{Z}:=$ $\mathscr{L}_{p}(\Omega, \mathcal{F}, P)$ and $\mathbb{Z}^{*}$ its dual. A real valued function $\rho: \mathbb{Z} \rightarrow \mathbb{R}_{+}$is called a coherent risk measure if it satisfies the axiom of risk measures including convexity, monotonicity, translation invariance and positive homogeneity, see Artzner et al. [3] for details. It is well known that if $\rho$ is a coherent risk measure, then $\rho(Z)$ can be written as

$$
\rho(Z)=\sup _{\zeta \in \mathfrak{A}} \mathbb{E}_{\zeta}[Z]
$$

where $\mathfrak{A}=\{\zeta \in \mathfrak{B}:\langle\zeta, Z\rangle \leq \rho(Z), \forall Z \in \mathbb{Z}\}$ and

$$
\mathfrak{B}:=\left\{\zeta \in \mathbb{Z}^{*}: \int_{\Omega} \zeta(\omega) d P(\omega)=1, \zeta \geq 0\right\}
$$

see comprehensive discussions by Shapiro et al. in [43, Chapter 6].

Let us now return to consider a stochastic Nash equilibrium where each player aims to minimize its risk

$$
\min _{y_{i} \in Y_{i}} \rho_{i}\left(f_{i}\left(y_{i}, y_{-i}, \xi\right)\right),
$$

where $f_{i}\left(y_{i}, y_{-i}, \xi\right)$ is player $i$ 's random objective function such as value of an asset or a portfolio loss. In the case when $\rho_{i}$ is a coherent risk measure, we can reformulate (3.11) as a DRNE based on the discussions above

$$
\min _{y_{i} \in Y_{i}} \sup _{\zeta \in \mathfrak{A}} \mathbb{E}_{\zeta}\left[f_{i}\left(y_{i}, y_{-i}, \xi\right)\right]
$$


In what follows, we consider two particular coherent risk measures: coherent entropic risk measure and conditional value at risk ( $\mathrm{CVaR}$ for short). Since both of them are closely related to tail distribution of the underlying random variable, they are also known as tail risk measures.

1. Stochastic Nash equilibrium with coherent entropic risk measure. Let $Z \in \mathbb{Z}$ be a random variable. The entropic risk measure of $Z$ is defined as

$$
e_{\gamma}(Z):=\frac{1}{\gamma} \ln \mathbb{E}_{P}\left[e^{-\gamma Z}\right]
$$

where $\gamma$ is a positive constant. Note that $e_{\gamma}(\cdot)$ is a convex risk measure but not necessarily coherent [18]. Föllmer and Knispel [19] develop a coherent version of the entropic risk measure by considering the Moreau-Fenchel duality of $e_{\gamma}(\cdot)$

$$
\rho_{\tau}(Z):=\inf _{\gamma>0} \frac{\tau}{\gamma}+e_{\gamma}(Z)
$$

where $\tau>0$ is a parameter. An important feature of $\rho_{\tau}(\cdot)$ is that

$$
\rho_{\tau}(Z)=\sup _{P \in \mathcal{P}_{\tau}} \mathbb{E}_{P}[-Z]
$$

where $\mathcal{P}_{\tau}:=\left\{Q \in \mathscr{P}(\Xi): \mathbb{D}_{\mathrm{KL}}(Q, P) \leq \tau\right\}, \tau$ is a positive number and $\mathbb{D}_{\mathrm{KL}}(\cdot, \cdot)$ denotes the Kullback-Leibler (KL for short) divergence. In the literature on distributionally robust optimization, $P$ is known as a nominal distribution which may be constructed through empirical data. KL divergence originates in the field of information theory, and it has been widely used in the area of the distributionally robust optimization, see $[20,22,30]$ for recent applications.

Substituting the coherent risk measure $\rho_{\tau}(\cdot)$ into (3.11), we obtain the following risk averse stochastic Nash equilibrium

$$
y_{i} \in \arg \min _{y_{i} \in Y_{i}} \rho_{\tau_{i}}\left(f_{i}\left(y_{i}, y_{-i}, \xi\right)\right), \text { for } i=1, \cdots, m
$$

and its DRNE formulation

$$
y_{i} \in \arg \min _{y_{i} \in Y_{i}} \sup _{P \in \mathcal{P}_{i}} \mathbb{E}_{P}\left[f_{i}\left(y_{i}, y_{-i}, \xi\right)\right] \text {, for } i=1, \cdots, m,
$$

where $\mathcal{P}_{i}:=\left\{Q \in \mathscr{P}: \mathbb{D}_{\mathrm{KL}}\left(Q, P_{i}\right) \leq \tau_{i}\right\}, P_{i}$ is player $i$ 's nominal distribution, $\tau_{i}$ is player $i$ 's ambiguity parameter. Moreover, by using [19, Proposition 3.1], we have

$$
\min _{y_{i} \in Y_{i}} \sup _{P \in \mathcal{P}_{i}} \mathbb{E}_{P}\left[f_{i}\left(y_{i}, y_{-i}, \xi\right)\right]=\min _{y_{i} \in Y_{i}, \gamma_{i}>0} \frac{\tau_{i}}{\gamma_{i}}+e_{\gamma_{i}}\left(-f_{i}\left(y_{i}, y_{-i}, \xi\right)\right) .
$$

By taking $\left(y_{i}, \gamma_{i}\right)$ as the decision variable of player $i$, we may consider the following Nash equilibrium:

$$
\left(y_{i}^{*}, \gamma_{i}^{*}\right) \in \arg \min _{y_{i} \in Y_{i}, \gamma_{i}>0} \frac{\tau_{i}}{\gamma_{i}}+e_{\gamma_{i}}\left(-f_{i}\left(y_{i}, y_{-i}^{*}, \xi\right)\right), \text { for } i=1, \cdots, m
$$


In what follows, we show that solving DRNE (3.12) is equivalent to solving (3.13). From a computational perspective, the latter is more preferable because it is an ordinary deterministic Nash equilibrium problem.

Proposition 3.1 $y^{*}$ is a Nash equilibrium of problem (3.12) if and only if there exists a $\gamma^{*}>0$ such that $\left(y^{*}, \gamma^{*}\right)$ solves $(3.13)$.

Proof. Following a similar argument to Proposition 2.1, we can show that $\left(y^{*}, \gamma^{*}\right)$ is a solution to (3.13) if and only if it solves the following optimization problem

$$
\min _{y \in Y, \gamma>0} \sum_{i=1}^{m} \frac{\tau_{i}}{\gamma_{i}}+e_{\gamma_{i}}\left(-f_{i}\left(y_{i}, y_{-i}^{*}, \xi\right)\right) .
$$

Let $\left(y^{*}, \gamma^{*}\right)$ be an optimal solution to problem (3.14). Suppose that $y^{*}$ is not a Nash equilibrium of (3.12), that is, there exists, $\bar{y} \in Y$ such that

$$
\sum_{i=1}^{m} \rho_{\tau_{i}}\left(-f_{i}\left(\bar{y}_{i}, y_{-i}^{*}, \xi\right)\right)<\sum_{i=1}^{m} \rho_{\tau_{i}}\left(-f_{i}\left(y_{i}^{*}, y_{-i}^{*}, \xi\right)\right) .
$$

Then

$$
\begin{aligned}
\min _{\gamma>0} \sum_{i=1}^{m} \frac{\tau_{i}}{\gamma_{i}}+e_{\gamma_{i}}\left(-f_{i}\left(\bar{y}_{i}, y_{-i}^{*}, \xi\right)\right) & =\sum_{i=1}^{m} \min _{\gamma_{i}>0} \frac{\tau_{i}}{\gamma_{i}}+e_{\gamma_{i}}\left(-f_{i}\left(\bar{y}_{i}, y_{-i}^{*}, \xi\right)\right) \\
& <\sum_{i=1}^{m} \min _{\gamma_{i}>0} \frac{\tau_{i}}{\gamma_{i}}+e_{\gamma_{i}}\left(-f_{i}\left(y_{i}^{*}, y_{-i}^{*}, \xi\right)\right) \\
& =\sum_{i=1}^{m} \frac{\tau_{i}}{\gamma_{i}^{*}}+e_{\gamma_{i}^{*}}\left(-f_{i}\left(y_{i}^{*}, y_{-i}^{*}, \xi\right)\right),
\end{aligned}
$$

which yields a contradiction to the optimality of $\left(y^{*}, \gamma^{*}\right)$. Conversely, if $y^{*}$ solves (3.12), that is, for any $\bar{y} \in Y$,

$$
\sum_{i=1}^{m} \rho_{\tau_{i}}\left(-f_{i}\left(\bar{y}_{i}, y_{-i}^{*}, \xi\right)\right) \geq \sum_{i=1}^{m} \rho_{\tau_{i}}\left(-f_{i}\left(y_{i}^{*}, y_{-i}^{*}, \xi\right)\right),
$$

then by [19, Proposition 3.1], there exists a positive $\gamma^{*}$ such that

$$
\begin{aligned}
\sum_{i=1}^{m} \min _{\gamma_{i}>0} \frac{\tau_{i}}{\gamma_{i}}+e_{\gamma_{i}}\left(-f_{i}\left(\bar{y}_{i}, y_{-i}^{*}, \xi\right)\right) & \geq \sum_{i=1}^{m} \min _{\gamma_{i}>0} \frac{\tau_{i}}{\gamma_{i}}+e_{\gamma_{i}}\left(-f_{i}\left(y_{i}^{*}, y_{-i}^{*}, \xi\right)\right) \\
& =\sum_{i=1}^{m} \frac{\tau_{i}}{\gamma_{i}^{*}}+e_{\gamma_{i}^{*}}\left(-f_{i}\left(y_{i}^{*}, y_{-i}^{*}, \xi\right)\right),
\end{aligned}
$$

which means that $\left(y^{*}, \gamma^{*}\right)$ is an optimal solution to problem (3.14).

2. Stochastic Nash equilibrium with conditional value at risk (CVaR). Conditional value at risk, sometimes called expected shortfall has received a great deal of attention as a 
measure of risk $[38,53]$. In a financial context, it has a number of advantages over the commonly used value at risk (VaR) and has been proposed as the primary tool for banking capital regulation in the draft Basel III standard.

For a given random variable $Z \in \mathbb{Z}$ and confidence level $\alpha \in(0,1)$, CVaR is defined as

$$
\operatorname{CVaR}_{\alpha}(Z):=\frac{1}{1-\alpha} \int_{\left\{\xi: Z(\xi) \geq \operatorname{VaR}_{\alpha}(Z)\right\}} Z P(d \xi),
$$

where $\operatorname{VaR}_{\alpha}(Z):=\inf \{t: F(t) \geq \alpha\}$ and $F(\cdot)$ is the cumulative distribution function of $Z$.

Assuming that each player is risk averse by minimizing the CVaR risk measure, then we arrive at a stochastic Nash equilibrium model with CVaR risk measure

$$
y_{i} \in \arg \min _{y_{i} \in Y_{i}} \operatorname{CVaR}_{\alpha_{i}}\left(f_{i}\left(y_{i}, y_{-i}, \xi\right)\right) \text {, for } i=1, \cdots, m \text {. }
$$

By the dual representation [43, Chapter 6], (3.15) can be recast into a DRNE

$$
y_{i} \in \arg \min _{y_{i} \in Y_{i}} \sup _{P \in \mathcal{P}_{i}} \mathbb{E}_{P}\left[f_{i}\left(y_{i}, y_{-i}, \xi\right)\right] \text {, for } i=1, \cdots, m,
$$

where

$$
\mathcal{P}_{i}:=\left\{P \in \mathscr{P}: g_{P}(\xi) \in\left[0, \frac{1}{1-\alpha_{i}}\right], \xi \in \Xi\right\},
$$

and $g_{P}(\cdot)$ denotes the density function corresponding to $P$. Moreover, by Rockafellar and Uryasev [38, Theorem 1], minimizing CVaR can be achieved by minimizing a more tractable auxiliary function without predetermining the corresponding VaR first, that is,

$$
\mathrm{CVaR}_{\alpha}(Z)=\min _{\eta \in \mathbb{R}} \eta+\frac{1}{1-\alpha} \int_{\Xi}(Z-\eta)_{+} P(d \xi),
$$

where $(t)_{+}=\max (0, t)$. Then we may reformulate the DRNE as:

$$
\left(y_{i}^{*}, \eta_{i}^{*}\right) \in \arg \min _{y_{i} \in Y_{i}, \eta_{i}} \psi_{i}\left(y_{i}, y_{-i}^{*}, \eta_{i}\right), \text { for } i=1, \cdots, m,
$$

where

$$
\psi_{i}\left(y_{i}, y_{-i}^{*}, \eta_{i}\right):=\eta_{i}+\frac{1}{1-\alpha_{i}} \int_{\xi \in \Xi}\left(f_{i}\left(y_{i}, y_{-i}^{*}, \xi\right)-\eta_{i}\right)_{+} P(d \xi) .
$$

Similar to Proposition 3.1, we have the following result.

Proposition 3.2 $y^{*}$ solves DRNE (3.16) if and only if there exists an $\eta^{*}$ such that $\left(y^{*}, \eta^{*}\right)$ solves (3.17). 


\section{Distributionally robust Stackelberg models}

The DRNE model that we discussed in the preceding sections are restricted to the case that players are in an equal competitive status under Nash conjecture. In this section, we deviate from the model by considering a hierarchical structure namely Stackelberg leader-follower model to describe a situation where some players are in a more strategic position than others such as new entrants of a market or capacity expansion of a dominant player. Note that research on stochastic Stackelberg model has been well documented, see for example De Wolf and Smeers [15] and DeMiguel and Xu [14]. Here we consider a robust counterpart where information on the underlying uncertainty is incomplete. Specifically we consider two models: (a) Stackelberg distributionally robust follower model where the leader is risk neutral and/or has more information about the underlying uncertainty whereas the followers have less information and hence are more risk averse, (b) Stackelberg distributionally robust leader model where leader is risk averse by playing robust strategy and followers play a stochastic game.

\subsection{Stackelberg robust followers model}

Let us start by considering a situation where the leader is interested in the equilibrium outcome of the followers and it has some mechanism to influence the equilibrium to achieve its own goal. In practice, the leader could be a strategic market player who plans to enter a new market or expand its existing capacity, it can also be a regulator of a new market. We will come to concrete applications in Section 5.

Let $z$ denote the decision vector of the leader and $h(z, y)$ its utility function. The utility function depends on $z$ and the equilibrium of the followers is denoted by $y$. The leader presumes that the followers are risk averse due to incomplete information on the distribution of the underlying uncertainty and hence they play a distributionally robust game:

$$
\min _{y_{i} \in Y_{i}} \max _{P \in \mathcal{P}_{i}} \mathbb{E}_{P}\left[f_{i}\left(z, y_{i}, y_{-i}, \xi\right)\right] \text {, for } i=1, \cdots, m \text {. }
$$

The dependence of $f_{i}$ on $z$ means that the leader's decision has an impact on the disutility of the followers.

We consider the leader's optimal decision making problem which is to find an optimal decision $z$ which maximizes its utility $h(z, y)$ with anticipation that the followers will reach an equilibrium (4.18), that is,

$$
\begin{array}{cl}
\max _{z \in Z, y} & h(z, y) \\
\text { s.t. } & y \in S(z),
\end{array}
$$

where $S(z)$ denotes the set of Nash equilibria of (4.18) for each given $z \in Z$. In the case when 
$S(z)$ is not a singleton, formulation (4.19) means that the leader's optimal decision is based on the best equilibrium in favor of the leader. We say this is an optimistic model. A pessimistic model can be formulated if we consider the worst equilibrium of the followers:

$$
\begin{array}{lll}
\max _{z \in Z} & \min _{y} & h(z, y) \\
& \text { s.t. } & y \in S(z) .
\end{array}
$$

The two models coincide when the set $S(z)$ reduces to a singleton, see similar discussions by Shapiro and $\mathrm{Xu}[44$, Section 2] on a stochastic Stackelberg model.

In what follows, we discuss numerical schemes for solving problem (4.19). Assuming

$$
\max _{P \in \mathcal{P}_{i}} \mathbb{E}_{P}\left[f_{i}\left(z, y_{i}, y_{-i}, \xi\right)\right]
$$

is convex in $y_{i}$. Then each player's optimization problem can be represented by its first-order Karush-Kuhn-Tucker condition. The main challenge here is that the function is not necessarily continuously differentiable.

Recall that Clarke subdifferential of a locally Lipschitz continuous function $\phi(x)$ at $x$, denoted by $\partial \phi(x)$, is defined as follows:

$$
\partial \phi(x):=\operatorname{conv}\left\{\lim _{x^{\prime} \in D, x^{\prime} \rightarrow x} \nabla \phi\left(x^{\prime}\right)\right\}
$$

where $D$ denotes the set of points near $x$ at which $\phi$ is Fréchet differentiable, and $\nabla \phi(x)$ denotes the gradient of $\phi$ at $x$. For a convex set $C \subseteq \mathbb{R}^{n}$, the normal cone to $x$ at $x \in C$ is

$$
\mathcal{N}_{C}(x)=\left\{\zeta \in \mathbb{R}^{n}: \zeta^{T} d \leq 0, \forall d \in \mathcal{T}_{C}(x)\right\}
$$

where $\mathcal{T}_{C}(x)=\liminf _{t \rightarrow 0, C \ni x^{\prime} \rightarrow x} \frac{1}{t}\left(C-x^{\prime}\right)$ and $\mathcal{N}_{C}(x)=\emptyset$ when $x \notin C$; see [11] for details of the subdifferential and normal cone.

Assume that for fixed $\left(z, y_{-i}, \xi\right), f_{i}\left(z, y_{i}, y_{-i}, \xi\right)$ is convex w.r.t. $y_{i}$ and $Y_{i}$ is a convex set and denote

$$
\vartheta_{i}\left(z, y_{i}, y_{-i}\right):=\max _{P \in \mathcal{P}_{i}} \mathbb{E}_{P}\left[f_{i}\left(z, y_{i}, y_{-i}, \xi\right)\right]
$$

Then, $\vartheta_{i}\left(z, y_{i}, y_{-i}\right)$ is convex in $y_{i}$ for each given $z$ and $y_{-i}$. Subsequently, the robust Nash equilibrium is equivalent to

$$
0 \in \partial_{y_{i}} \vartheta_{i}\left(z, y_{i}, y_{-i}\right)+\mathcal{N}_{Y_{i}}\left(y_{i}\right), \text { for } i=1, \cdots, m,
$$

where $\mathcal{N}_{Y_{i}}\left(y_{i}\right)$ is the normal cone to $Y_{i}$ at $y_{i} \in Y_{i}$. Consequently, (4.19) can be equivalently written as

$$
\begin{array}{ll}
\min _{z, y} & h(z, y) \\
\text { s.t. } & z \in Z \\
& 0 \in \partial_{y_{i}} \vartheta_{i}\left(z, y_{i}, y_{-i}\right)+\mathcal{N}_{Y_{i}}\left(y_{i}\right), \text { for } i=1, \cdots, m .
\end{array}
$$


The equilibrium constraint is difficult to handle since $\partial_{y_{i}} \vartheta_{i}\left(z, y_{i}, y_{-i}\right)$ is a set-valued mapping. However, when $\vartheta_{i}\left(z, y_{i}, y_{-i}\right)$ is continuously differentiable w.r.t. $y_{i},(4.20)$ reduces to an ordinary MPEC which can be solved by available code such as NLPEC (in GAMS).

Example 4.1 (KL divergence) Suppose that follower $i$ does not have complete information on the distribution of uncertainty $\xi$ and it constructs an estimation $P_{i}$ based on some historical information. For safety, it takes the ambiguity set

$$
\mathcal{P}_{i}:=\left\{Q \in \mathscr{P}: \mathbb{D}_{\mathrm{KL}}\left(Q, P_{i}\right) \leq \tau_{i}\right\}
$$

where $\tau_{i}>0$ is the ambiguity (or robust) parameter. By the analysis in the section 3.2, we may reformulate the follower's Nash equilibrium (4.18) as:

$$
y_{i}^{*} \in \arg \min _{y_{i} \in Y_{i}} \rho_{\tau_{i}}\left(f_{i}\left(z, y_{i}, y_{-i}^{*}, \xi\right)\right), \text { for } i=1, \cdots, m .
$$

Suppose that $f_{i}(\cdot, \xi)$ is continuously differentiable for every $\xi \in \Xi$. Then by proposition 3.1 , we may reformulate the distributionally robust follower model as:

$$
\begin{array}{ll}
\min _{z, y, \tau} & h(z, y) \\
\text { s.t. } & z \in Z, \\
& 0 \in \nabla_{y_{i}} \rho_{\tau_{i}}\left(z, y_{i}, y_{-i}, \gamma_{i}\right)+\mathcal{N}_{Y_{i}}\left(y_{i}\right), \text { for } i=1, \cdots, m, \\
& \nabla_{\gamma_{i}} \rho_{\tau_{i}}\left(z, y_{i}, y_{-i}, \gamma_{i}\right)=0, \text { for } i=1, \cdots, m,
\end{array}
$$

which is a standard stochastic MPEC.

Example 4.2 (Moment information) Consider the case that followers only have some moment information and they construct the ambiguity sets as in (3.6). Suppose the conditions of Example 3.1 hold, and then for any given leader's decision $z$, the follower's DRNE model is:

$$
y_{i}^{*} \in \arg \min _{y_{i} \in Y_{i}} f_{i}\left(z, y_{i}, y_{-i}^{*}, \mu\right), \text { for } i=1, \cdots, m
$$

where $\mu$ is the mean value of random variable $\xi$. If in addition, $f_{i}(\cdot, \mu), i=1, \cdots, m$, is continuously differentiable, we may reformulate the distributionally robust follower model as:

$$
\begin{array}{ll}
\min _{z, y} & h(z, y) \\
\text { s.t. } & z \in Z, \\
& 0 \in \nabla_{y_{i}} f_{i}\left(z, y_{i}, y_{-i}, \mu\right)+\mathcal{N}_{Y_{i}}\left(y_{i}\right), \text { for } i=1, \cdots, m,
\end{array}
$$

which is an MPEC.

Similarly, if the followers construct the ambiguity sets in the form of (3.7) based on the moment information. Then under the conditions of Example 3.2 and continuously differentiable 
assumption, we may reformulate the distributionally robust follower model as:

$$
\begin{array}{ll}
\min _{z, y} & h(z, y) \\
\text { s.t. } & z \in Z, \\
& 0 \in \nabla_{y_{i}} \tilde{f}_{i}\left(z, y_{i}, y_{-i}\right)+\mathcal{N}_{Y_{i}}\left(y_{i}\right), \text { for } i=1, \cdots, m,
\end{array}
$$

where $\tilde{f}(\cdot)$ is defined in the form of $(3.8)$. Again, we reformulate the distributionally robust follower problem as an MPEC.

\subsection{Stackelberg robust leader model}

In the previous subsection, we consider robust follower model in the framework of Stackelberg paradigm. We now turn to discuss robust leader model under the same framework where the followers play a stochastic Nash game. This applies to practice such as introduction of a new market where the regulator (the leader) contemplates outcome of competition of market players (followers). In that case, the leader usually has less information about the underlying extraneous uncertainty whereas the followers will have complete information about the distribution of the uncertainty when the game is played, that is, for each $P \in \mathcal{P}$ and leader's variable $z \in Z$, the follower's problem can be formulated as a stochastic Nash equilibrium:

$$
y_{i}^{*} \in \arg \min _{y_{i} \in Y_{i}} \mathbb{E}_{P}\left[f_{i}\left(z, y_{i}, y_{-i}^{*}, \xi\right)\right], \text { for } i=1, \cdots, m \text {. }
$$

Assume that the leader (regulator) is able to construct a set of probability distributions $\mathcal{P}$ based on incomplete information and believes the true probability distribution lies in the set. Then the leader would contemplate all possible equilibrium from each of the distributions and base his optimal decision on the worst equilibrium outcome to hedge the risks. Mathematically, the leader solves the following optimization problem

$$
\min _{z \in Z} \max _{P \in \mathcal{P}} \max _{y(z, P) \in S(z, P)} h(z, y(z, P))
$$

where $S(z, P)$ denotes the set of Nash equilibrium of (4.21). If in addition, for each $z \in Z$, $f_{i}\left(z, y_{i}, y_{-i}, \xi\right)$ is convex w.r.t. $\left(y_{i}, y_{-i}\right)$ and $Y_{i}$ is a convex set. We can reformulate (4.22) as

$$
\begin{aligned}
& \min _{z \in Z} \max _{P \in \mathcal{P}, y \in Y} h(z, y) \\
& \text { s.t. } \quad 0 \in \mathbb{E}_{P}\left[\nabla_{y_{i}} f_{i}\left(z, y_{i}, y_{-i}, \xi\right)\right]+\mathcal{N}_{Y_{i}}\left(y_{i}\right) \text {, for } i=1, \cdots, m \text {. }
\end{aligned}
$$

Problem (4.23) is a kind of robust formulation of mathematical program with stochastic variational inequality constraint. However, it differs from standard formulation of DRO model in that the inner maximization problem takes not only the worst probability distribution from ambiguity set $\mathcal{P}$ but the worst equilibrium of the followers. Moreover, the maximization in $P$ 
and $y$ cannot be separated which means we are unable to derive a dual formulation of the maximization w.r.t. $P$, a standard approach in DRO. However, when follower's problem has some specific structure, then problem (4.23) can be converted into a standard DRO. The following proposition states that.

Proposition 4.1 Suppose that (a) for fixed leader's decision $z$, leader's object $h(z, y)$ is linear in $y$; (b) for each given leader's decision $z$ and probability measure $P \in \mathcal{P}$, there exists a unique stochastic Nash equilibrium $y(z, P)=\mathbb{E}_{P}[g(z, \xi)]$, where $g(z, \xi)$ is a measure function. Then problem (4.22) can be reformulated as a standard DRO:

$$
\min _{z \in Z} \max _{P \in \mathcal{P}} \mathbb{E}_{P}[h(z, g(z, \xi))]
$$

We omit the proof as it is straightforward. Here we give an example to explain the claim.

Example 4.3 Consider the case where the leader is a regulator who chooses its decision variable $z \in Z$ to affect the follower's plan. Assume for simplicity that there are two followers who compete to provide homogeneous goods or services to a market. Market demand is described by an inverse demand function

$$
p(q, z, \xi)=a(z, \xi)-b(z, \xi) q
$$

where $q$ is the total supply to the market and $\xi$ is a random variable representing uncertainty in demand. The dependence on $z$ means that the regulator has an influence on the demand, i.e., tax on goods or services. Let $x_{1}$ and $x_{2}$ denote the production by firm 1 and firm 2. Assume that both firms need to make a decision on their production level prior to the realization of demand uncertainty and they can observe decisions from each other. Then firm 1's decision making problem is

$$
\begin{array}{ll}
\max _{x_{1}} & \mathbb{E}_{P}\left[\left(a(z, \xi)-b(z, \xi)\left(x_{1}+x_{2}\right)\right) x_{1}\right]-c_{1} x_{1} \\
\text { s.t. } & 0 \leq x_{1} \leq u_{1}
\end{array}
$$

where $c_{1} x_{1}$ is the production cost and $u_{1}$ is the capacity limit. Likewise, we can formulate firm 2's decision problem

$$
\begin{array}{ll}
\max _{x_{2}} & \mathbb{E}_{P}\left[\left(a(z, \xi)-b(z, \xi)\left(x_{1}+x_{2}\right)\right) x_{2}\right]-c_{2} x_{2} \\
\text { s.t. } & 0 \leq x_{2} \leq u_{2} .
\end{array}
$$

Assume for the simplicity of discussion that $u_{1}$ and $u_{2}$ are sufficiently large such that at any equilibrium neither firm will reach its capacity limit. Suppose also that for any $z$ and $P \in \mathcal{P}$, 
$\mathbb{E}[b(z, \xi)]>0$ (a sufficient condition is that $b(z, \xi)>0, \forall z \in Z, \xi \in \Xi$ ) and $c_{1}, c_{2}>0$. Then by [46, Theorem 1], there exists a unique Nash equilibrium:

$$
\left\{\frac{\mathbb{E}_{P}[a(z, \xi)]+c_{2}-2 c_{1}}{\mathbb{E}_{P}[3 b(z, \xi)]}, \frac{\mathbb{E}_{P}[a(z, \xi)]+c_{1}-2 c_{2}}{\mathbb{E}_{P}[3 b(z, \xi)]}\right\}
$$

Suppose that the leader's utility is a linear function $f(z, y)$ in the follower's response $y$ and $b(z, \xi)=b(z)$ is not dependent on $\xi$, and then the leader's problem can be reformulated as:

$$
\min _{z \in Z} \max _{P \in \mathcal{P}} \mathbb{E}_{P}\left[h(z, y):=h\left(z,\left(\frac{a(z, \xi)+c_{2}-2 c_{1}}{3 b(z)}, \frac{a(z, \xi)+c_{1}-2 c_{2}}{3 b(z)}\right)\right)\right],
$$

which is a standard DRO problem.

\section{Hierarchical capacity competition problem}

Supply chain and outsourcing practices are ubiquitous in today's competitive global business environment. In the capacity investment context, we have seen that for entering a new, uncertain market, a firms may not only invests in its own capacity but also outsources a certain amount of production to other firms who need to expand their capacity, as well, for obtaining the orders. A notable case in semiconductor manufacturing is that the global giant Samsung always expands its capacity and outsources to other competing manufacturers in China, Japan and Taiwan at the same time. In consumer electronics sector, the similar strategy is also found in Apple's new product launches such as the next generations of iPhone and iPad.

This distinctive business practice of simultaneous capacity investment and outsourcing in high-tech sectors motivates us to study a supply chain model of hierarchical capacity competition where a buying firm (buyer; e.g., Samsung) not only invests in its own capacity to supply a new, uncertain market but also outsources a certain amount of market supply to multiply compete with supplying firms (suppliers, $i=1, \cdots, m$; e.g., semiconductor manufacturers in China, Japan and Taiwan) who invest in their capacity for the buyer's orders (that can be thought of as a spot market).

We suppose that the buyer and the suppliers follow the following rules: the buyer and suppliers sign a forward contract with fixed qualities and strike price $a_{0} p_{b}$, where $0<a_{0}<1$ is parameter and $p_{b}$ is buyer's unit price determined by the market. The suppliers must fulfil the contract with the buyer and sell the residual production directly to the market. Likewise, the supplier's market price $p_{i}^{s}$ is determined by market, which is often lower than buyer's market price. The buyer's demand of end-product to consumers satisfies the following stochastic linear inverse demand function:

$$
p_{b}(x, y, z, \xi)=a_{b}-r_{b}\left(x+\sum_{i=1}^{m} y_{i}\right)-r_{b}^{s} \sum_{i}^{m}\left(z_{i}-y_{i}\right)+\xi,
$$


where $a_{b}$ is the upper bound for customers' willingness to pay for the buyer's product, $\xi$ is a random variable which characterizes the fluctuation of the price, $r_{b}$ is buyer's inverse demand sensitivity, $r_{b}^{s}$ is suppliers's inverse demand sensitivity, $x$ is the buyer's supply via its own capacity, $y_{i}$ is the buyer's supply via the supplier $i$ 's production, and $z_{i}$ is the total capacity of supplier $i$. For simplicity, we denote $Y=\sum_{i=1}^{m} y_{i}$ and $Z=\sum_{i=1}^{m} z_{i}$. Then

$$
p_{b}(x, y, z, \xi)=a_{b}-r_{b}(x+Y)-r_{b}^{s}(Z-Y)+\xi
$$

The buyer's ex ante profit can be written as

$$
\pi_{b}(x, y, z, \xi)=\left[p_{b}(x, y, z, \xi)-c_{b}\right](x+Y)-v_{b} x-a_{0} p_{b}(x, y, z, \xi) Y,
$$

where $c_{b}$ is the buyer's marginal cost of end-product production, $v_{b}$ is the buyer's marginal investment cost of capacity.

We suppose that the buyer knows how the suppliers will choose their decisions (see the discussion below) but does not know which demand scenario will occur in future at the time when is made the decision. As being a dominant player over other players, we suppose that buyer has more information on $\xi$ and it is a risk taker. Then the buyer can do at best is to maximize the expected profit based on its knowledge on the market demand. For simplicity, we consider the case that the buyer has full information of random $\xi$. Then buyer's ex post decision problem can be formulated as

$$
\begin{array}{cl}
\max _{x \geq 0, y \geq 0, z \geq 0} & \mathbb{E}_{P}\left[\pi_{b}(x, y, z, \xi)\right] \\
\text { s.t. } & z \in S(x, y),
\end{array}
$$

where $S(x, y)$ denotes the set of suppliers' optimal decision corresponding to the given buyer's decision $(x, y)$. Similar to problem (4.19), model (5.24) may be interpreted as an optimistic view of the buyer if $S(x, y)$ is not singleton.

Next, we analyze the suppliers' optimal decision. Given the buyer's capacity $x$ and order quality $y$, the $i$ th supplier chooses its level of capacity investment, $z_{i}$, to maximize its profit from selling its production to the buyer and market. Suppose that supplier $i$ 's demand of end-product to consumers satisfies the similar stochastic linear inverse demand function:

$$
p_{i}^{s}(x, y, z, \xi)=a_{i}^{s}-r_{b}(x+Y)-r_{b}^{s}(Z-Y)+\xi,
$$

where $a_{i}^{s}$ is the upper bound for customers' willingness to pay for the supplier $i$ 's product. We should keep in mind that $a_{i}^{s}<a_{b}$ as supplier is the small player which means its value of brand is lower than buyer's. For the given buyer's decision $(x, y)$ and other suppliers decision $Z_{-i}$, the $i$ th supplier's ex post profit is:

$$
\pi_{i}^{s}\left(z_{i}, \xi\right)=\left[a_{0} p_{b}(x, y, z, \xi)-r_{i}\right] y_{i}+\left[p_{i}^{s}(x, y, z, \xi)-c_{i}\right]\left(z_{i}-y_{i}\right)-v_{i} z_{i}
$$


where $r_{i}$ is the supplier $i$ 's marginal cost of component production, $c_{i}$ is the supplier $i$ 's marginal cost of end-product production, and $v_{i}$ is its marginal investment cost of capacity. Note that $r_{i}$ is different from $r_{b}$ so the quality difference between the buyer and suppliers' end products is considered.

For the given buyer's decision $(x, y)$ and other suppliers' decision $Z_{-i}$, supplier $i$ 's ex ante decision problem can be formulated as

$$
\begin{array}{cl}
\max _{z_{i}} & \mathbb{E}_{P}\left[\pi_{i}^{s}\left(z_{i}, \xi\right)\right] \\
\text { s.t. } & z_{i} \geq y_{i},
\end{array}
$$

where the constraints mean that the supplier's capacity must be no less than the order quality on the signed contract.

Different from the buyer, the suppliers may only have some partial information on $\xi$ and as a dominated players, they tend to be risk averse. Thus we may model them by DRNE, that is, by viewing the buyer's decision $(x, y)$ as given, the suppliers compete by playing a distributionally robust Nash-Cournot game:

$$
\max _{z_{i} \geq y_{i}} \inf _{P \in \mathcal{P}_{i}} \mathbb{E}_{P}\left[\pi_{i}^{s}\left(z_{i}, \xi\right)\right]
$$

where $\mathcal{P}_{i}$ is the ambiguity set perceived by supplier $i$ based on its information on the uncertainty. Obviously, for given $(x, y)$ and $Z_{-i}, \pi_{i}^{s}(\cdot, \xi)$ is a concave function. By Theorem 2.1, there is a robust Nash equilibrium, hence, $S(x, y) \neq \emptyset$.

In what follows, we discuss conditions for the uniqueness of equilibrium because the uniqueness is of interest from both computational and economic perspectives. We proceed the analysis by invoking Rosen's earlier result, namely [40, Theorem 2] for the uniqueness of a deterministic Nash equilibrium problem. Define

$$
\sigma(z, v)=\sum_{i=1}^{m} v_{i} \inf _{P \in \mathcal{P}_{i}} \mathbb{E}_{P}\left[\pi_{i}^{s}\left(z_{i}, \xi\right)\right],
$$

where $v=\left(v_{1}, \cdots, v_{m}\right) \geq 0$. According to [40, Theorem 2], if $\sigma(z, v)$ is diagonally strictly concave for some $r>0$ (see [40, Defintion 1]), then there exists a unique Nash equilibrium. In this context, a sufficient condition for $\sigma(z, v)$ to be diagonally strictly concave is that the Jacobian of function

$$
\Psi(z, v):=\left(\begin{array}{c}
v_{1} \nabla_{z_{1}} \inf _{P \in \mathcal{P}_{i}} \mathbb{E}_{P}\left[\pi_{1}^{s}\left(z_{1}, \xi\right)\right] \\
\vdots \\
v_{m} \nabla_{z_{m}} \inf _{P \in \mathcal{P}_{i}} \mathbb{E}_{P}\left[\pi_{m}^{s}\left(z_{m}, \xi\right)\right]
\end{array}\right)
$$

is a negative definite matrix. To this end, we need to discuss sufficient conditions for continuous differentiability of $\mathcal{P}_{i}, \inf _{P \in \mathcal{P}_{i}} \mathbb{E}_{P}\left[\pi_{i}^{s}\left(z_{i}, \xi\right)\right]$ in $z_{i}$. This is beyond the focus of this paper, we refer 
interested readers to [43, Chapater 6], where the authors discussed differentiability of coherent risk measures.

However, we might consider simpler case as we discussed in Section 2.1 that if the ambiguity set is defined through moment conditions such as (3.6) (or (3.7)), then we can obtain a closed form for $\inf _{P \in \mathcal{P}_{i}} \mathbb{E}_{P}\left[\pi_{i}^{s}\left(z_{i}, \xi\right)\right]$. Since $\pi_{i}^{s}(\cdot)$ is linear in $\xi$, if $\mathcal{P}_{i}, i=1, \cdots, m$, is in the form of (3.6), then

$$
\Psi(z, v):=\left(\begin{array}{c}
v_{1} \nabla_{z_{1}} \pi_{1}^{s}\left(z_{1}, \mu\right) \\
\vdots \\
v_{m} \nabla_{z_{m}} \pi_{m}^{s}\left(z_{m}, \mu\right)
\end{array}\right)
$$

where $\mu$ denotes the mean of $\xi$. For $v=(1, \cdots, 1)$,

$$
\nabla \Psi(z, v):=\left(\begin{array}{cccc}
-2 r_{b}^{s} & -r_{b}^{s} & \cdots & -r_{b}^{s} \\
-r_{b}^{s} & -2 r_{b}^{s} & \cdots & -r_{b}^{s} \\
\vdots & \vdots & \ddots & \vdots \\
-r_{b}^{s} & -r_{b}^{s} & \cdots & -2 r_{b}^{s}
\end{array}\right) .
$$

Since $r_{b}^{s}>0$, it is easy to verify the negative definiteness of $\nabla \Psi(z, v)$ by the principal minors. Consequently, the uniqueness follows.

\subsection{Numerical study}

We consider the case that there is a buyer, $b$, and two suppliers, $i \in\{1,2\}$, who both employ the KL divergence to be risk averse:

$$
\mathcal{P}:=\left\{Q \in \mathscr{P}: \mathbb{D}_{\mathrm{KL}}\left(Q, P_{0}\right) \leq \tau\right\}
$$

where $P_{0}$ is the nominal distribution. By the analysis of Example 4.1, we may reformulate the buyer's model as an MPEC problem and then we call the solver NLPEC under GAMS installed in a PC with Windows XP operating system to solve the MPEC. We report the numerical results for comparing the robust model with stochastic model by varying the ambiguity parameter $\tau$ from 0.05 to 0.8 . In the stochastic model, the suppliers also have full information of the uncertainty $\xi$ as does the buyer. We set the parameters involved in the model (5.24): $a_{b}=0.8$, $r_{b}=1, r_{b}^{s}=0.7, c_{b}=0.001, v_{b}=0.0015, a_{0}=0.8, a_{1}^{s}=0.5, a_{2}^{s}=0.65, c_{1}=0.003, c_{2}=0.003$, $r_{1}=0.002, r_{2}=0.002, v_{1}=1.1 c_{1}, v_{2}=1.1 c_{2}$, and the nominal $P_{0}$ is normally distributed with mean $\mu=0$ and standard deviation $\sigma=0.1$.

Figures 1 and 2 illustrate the numerical results of the robust follower model (5.24) with a fixed sample size 30 in the sample average approximation method and by increasing the ambiguity 
parameter $\tau$ (where $\tau=0$ is corresponding to the SP model). To reduce the impact of the samples on the results, we generate 30 groups of the sample and then take the average of the optimal solutions and optimal values. Figure 1 shows that buyer's profit (red solid line) has a tendency of increasing in the ambiguity parameter $\tau$. On the contrary, the suppliers's profit (blue dash-dot line) has a tendency of decreasing in the ambiguity parameter $\tau$. Moreover, the total profit of a supply chain (the green dash line) is stable with the value around 0.15. Figure 2 depicts the variation trend of buyer's (red solid line) and suppliers' (blue dash-dot line) capacity investments. It shows that the capacity investments have similar trends with the profits in Figure 1. By increasing the ambiguity parameter $\tau$, the market is more uncertain to the suppliers and they are thus reluctant to invest in capacity. At the same time, the buyer will invest more in capacity.

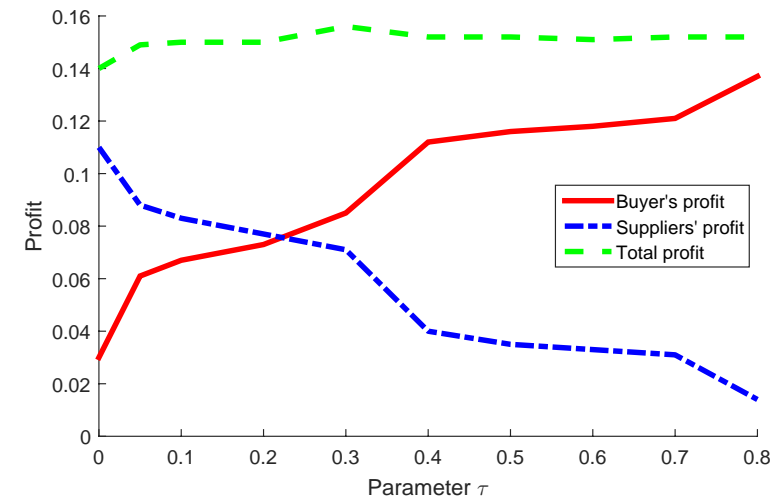

Figure 1: Capacity investment w.r.t ambiguity

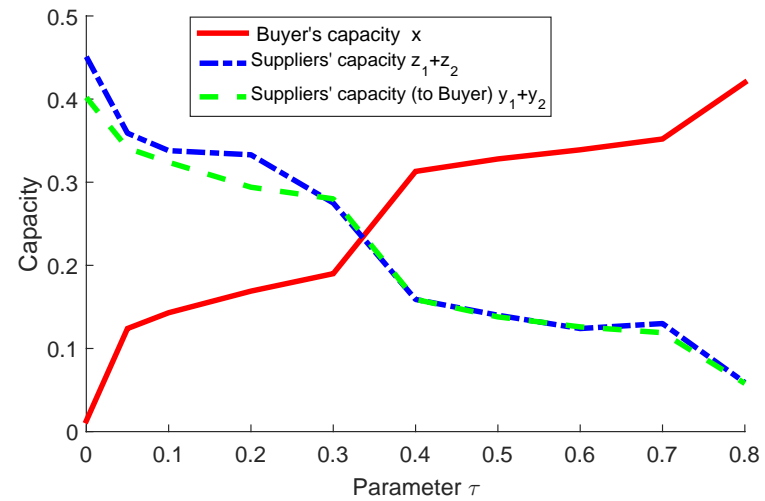

Figure 2: Profit w.r.t ambiguity parameter parameter

To summarize, our findings suggest that the suppliers' capacity investments and profits are increasing with the degree of informational completeness on demand uncertainty, but the buyer's capacity investment and profit are decreasing with the degree of informational completeness on demand uncertainty. In Figure 1, we can observe that the total supply chain profit remains unchanged over the value range of ambiguity parameter we consider. This is simply because the degree of information completeness on demand uncertainty primarily plays the role of how different supply-chain parties (buyer versus suppliers) appropriate the total profit they co-create, but does not impact the total profit generated by the entire supply chain. These observations point out an effective strategy for the buyer in a supply chain involving hierarchical capacity competition, consistent with the real-world practice: release less information on product demand to the suppliers so it can capture more supply chain profits by simultaneously investing heavily in the own capacity and outsourcing a sizable amount to the suppliers for utilizing most of their capacity. If the suppliers have more information on product demand, this strategy of 
simultaneous capacity investment and outsourcing is less effective since the buyer is harder to fully utilize the suppliers' capacity through outsourcing while they can also invest heavily in capacity to counteract with the buyer's strategic deployment. This numerical study shows the promise of the proposed methods contributing to a sizable body of operations and supply chain literature that many well-received insights are derived from stochastic Nash equilibrium schemes and they may be altered when decision makers have no complete information on underlying uncertainties, as demonstrated here.

\section{Concluding remarks}

Motivated by recent research on robust game theory and distributinally robust finite game $[1,36,31,32,2,41]$, we study several distributionally robust equilibrium models. We start with a distributionally robust Nash equilibrium (DRNE) model. Different from the existing studies, we focus on the general DRNE game rather than the finite DRNE games. We investigate the existence of equilibrium for the DRNE model, propose a numerical scheme for its computation, and specify some cases where the DRNE models can be reformulated as ordinary deterministic Nash equilibrium models. We then extend the discussion on DRNE to two distributionally robust Stackelberg models: a distributionally robust follower model and a distributionally robust leader model. Based on the discussion on DRNE, we reformulate the distributionally robust follower model as a mathematical program with equilibrium constraints and the distributionally robust leader model as a standard distributionally robust optimization problem under some moderate conditions. To demonstrate the applicability of our theoretical and numerical approaches, we employ the distributionally robust follower model to analyze the hierarchical capacity competition problem in a supply chain which, to the best of our knowledge, has not been systematically studied in the operations and supply chain literature.

It might be possible to take this work further in the following directions. First, it might be interesting to extend our model to the case where each player's objective is a distortion risk measure and explore existence of an equilibrium and computational schemes for calculating an equilibrium. Second, as we can see from this paper, the structure of the ambiguity set has a big impact on numerical solvability of our models. In the literature of DRO, a number of statistical methods have been proposed for construction of ambiguity set depending on the availability of information. It might be helpful to look into how our models and numerical schemes are played out when the ambiguity set of each player is constructed through Wasserstein metric. Moreover, in practice, the ambiguity set might be constructed through samples and other statistical information, and it will therefore be interesting to investigate how the change of information would affect the equilibrium. This kind of research has been carried out for DROs in the sense 
of stability analysis, see recent papers by Zhang et al. [54] and Sun and Xu [45]. It will be both theoretically and numerically interesting to establish qualitative and quantitative stability results for the robust equilibrium problems. Finally this paper touches briefly Stackelberg robust leader model where the leaders play a robust game whereas followers play a stochastic Nash game. We believe this kind of model is practically interesting but unfortunately here we don't have much space to explore the model and numerical scheme further.

Acknowledgements. We would like to thank the editor Professor Immanuel Bomze for organizing an effective review and two anonymous referees for insightful comments and constructive suggestions which help us significantly consolidate the paper.

\section{References}

[1] M. Aghassi and D. Bertsimas, Robust game theory, Math. Program., Vol. 107, pp. 231-273, 2006.

[2] S.D. Ahipasaogluy, R. Meskarianz, T. L. Magnantix and K. Natarajan, Beyond normality: a distributionally robust stochastic user equilibrium model, manuscript, 2014.

[3] P. Artzner, F. Delbaen, J-M. Eber and D. Heath, Coherent measures of risk, Math. Finance, Vol. 9, pp 203-228, 1999.

[4] B. Bank, J. Guddat, D. Klatte, B. Kummer and K. Tammer, Nonlinear Parametric Optimization, Akademine-Verlag, Berlin, 1982.

[5] A. Ben-Tal and E. Hochman, More bounds on the expectation of a convex function of a random variable. J. Appl. Prob., Vol. 9, pp. 803-812, 1972.

[6] A. Ben-Tal, L. El Ghaoui and A. Nemirovski, Robust Optimization, Princeton University Press, NJ, 2009.

[7] D. Bertsimas, D.B. Browny and C. Caramanis, Theory and applications of Robust Optimization, SIAM Rev., Vol 53. pp. 464-501, 2011.

[8] D. Bertsimas and I. Popescu, On the relation between option and stock prices: an optimization approach, Oper. Res., Vol. 50, pp. 358-374, 2002.

[9] J.F. Bonnans and A. Shapiro, Perturbation Analysis of Optimization Problems, Springer Series in Operations Research, Springer-Verlag, New York, 2000.

[10] G. Cachon and S. Netessine. 2004. Game theory in supply chain analysis. In Handbook of quantitative supply chain analysis: modeling in the e-business era, D. Simchi-Levi, S. D. 
Wu and M. Shen, editors, Kluwer Academic Publishers, 13-66. Reprinted in INFORMS Tutorials in Operations Research 2006, P. Grey, Series Editor, p. 200-233.

[11] F.H. Clarke, Optimization and Nonsmooth Analysis, Wiley, New York, 1983.

[12] E. Delage and Y. Ye, Distributionally robust optimization under moment uncertainty with application to data driven problems, Oper. Res., Vol. 58, pp. 595-612, 2010.

[13] E. Delage, S. Arroyo and Y. Ye, The Value of stochastic modeling in two-stage stochastic programs with cost uncertainty, Oper. Res., Vol. 62, pp. 1377-1393, 2014.

[14] V. DeMiguel and H. Xu, A stochastic multiple leader Stackelberg model: analysis, computation, and application, Oper. Res., Vol. 57, pp. 1220-1235, 2009.

[15] D. De Wolf, Y. Smeers, The gas transmission problem solved by an extension of the simplex algorithm, Manag. Scien., Vol. 46, pp. 1454-1465, 2000.

[16] P.M. Esfahani and D. Kuhn, Data-driven distributionally robust optimization using the Wasserstein metric: oerformance guarantees and tractable reformulations, Manuscript, 2015 .

[17] F. Facchinei and C. Kanzow, Generalized Nash equilibrium problems, 4OR, Vol. 5, pp. 173-180, 2007.

[18] H. Föllmer And A. Schied, Stochastic Finance: An Introduction in Discrete Time, Walter de Gruyter, Berlin, 2004.

[19] H. Föllmer and T. Knispel, Entropic risk measures: coherence vs. convexity, model ambiguity, and robust large deviations, Sto. Dynam., Vol. 11, pp. 333-351, 2011.

[20] Z. Hu and J. Hong, Kullback-Leibler divergence constrained distributionally robust optimization, manuscript, 2013.

[21] H. Jiang, S. Netessine and S. Savin, Robust newsvendor competition under asymmetric information, Oper. Res., Vol. 59, pp. 254-261, 2011.

[22] R. Jiang and Y. Guan, Data-driven chance constrained stochastic program, Math. Prog., to appear, 2015

[23] G.A. Hanasusanto, D. Kuhn and W. Wiesemann, K-adaptability in two-stage distributionally robust binary programming, manuscript, 2015.

[24] A. Haurie, G. Zaccour and Y. Smeers, Stochastic equilibrium programming for dynamic oligopolistic markets, J. Optim. Theory Appl., Vol. 66, pp. 243-253, 1990. 
[25] R. Henerion and W. Römisch, On M-stationary point for a stochastic equilibrium problem under equilibrium constraints in electricity spot market modeling, Appl. Math., Vol. 52, pp. 473-494, 2007.

[26] S. Kakutani, A generalization of Brouwer's fixed point theorem, Duke Mathematical Journal, Vol. 8, pp. 457-459, 1941.

[27] M. Kapsos, N. Christofides and B. Rustem, Worst-case robust Omega ratio, Eur. J. Oper. Res., Vol. 243, pp. 499-507, 2014.

[28] E. Kardes, F. Ordonez and E.W. Hall, Discounted robust stochastic games and an application to queuing control, Oper. Res., Vol. 59, pp. 365-382, 2011.

[29] A. Ling, J. Sun, N. Xiu and X. Yang, Robust two-stage stochastic linear optimization with risk aversion, Eur. J. Oper. Res., Vol. 256, pp. 215-229, 2017.

[30] Y. Liu and H. Xu, Entropic approximation for mathematical programs with robust equilibrium constraints. SIAM J. Optim., Vol. 24, pp. 933-958, 2014.

[31] N. Loizou, Distributionally robust game theory, arXiv preprint:1512.03253, 2015.

[32] N. Loizou, Distributionally robust games with risk-averse players, In Proceedings of 5th the International Conference on Operations Research and Enterprise Systems, pages 186-196, 2016.

[33] A. Pichler and H. Xu, Quantitative stability analysis for minimax distributionally robust risk optimization, Optimization online, January 2017.

[34] G.C. Pflug and D. Wozabal, Ambiguity in portfolio selection. Quant. Financ., Vol. 7, pp. 435-442, 2007.

[35] K. Postek, A. Ben-Tal, D. Hertog and B. Melenberg, Exact robust counterparts of ambiguous stochastic constraints under mean and dispersion information, manuscript, 2015.

[36] S.J. Qu and M. Goh, Distributionally robust games with an application to supply chain, manuscript, 2012.

[37] D. Ralph and Y. Smeers, Pricing risk under risk measures: an introduction to stochasticendogenous equilibria, manuscript, 2011.

[38] R.T. Rockafellar and S. Uryasev, Optimization of conditional value-at-risk, J. Risk, Vol. 2, pp. 21-41, 2000.

[39] U. Ravat and U.V. Shanbhag, On the characterization of solution sets of smooth and nonsmooth stochastic Nash games, SIAM J. Optim., Vol. 21, pp. 1168-1199, 2011. 
[40] J.B. Rosen, Existence and uniqueness of equilibrium point for concave n-person games, Econometrica, Vol. 33, pp. 520-534, 1965.

[41] V.V. Singh, O. Jouini, A. Lisser, Existence of Nash equilibrium for chance-constrained games, Oper. Res. Lett., Vol. 44, pp. 640-644, 2016.

[42] V.V. Singh, O. Jouini, A. Lisser, Distributionally robust chance-constrained games: Existence and characterization of Nash equilibrium, to appear in Optimization Letters.

[43] A. Shapiro, D. Dentcheva and A. Ruszczynski, Lectures on Sochastic Programming: Modeling and Theory, SIAM, Philadelphia, 2009.

[44] A. Shapiro and H. Xu, Stochastic mathematical programs with equilibrium constraints, modeling and sample average approximation, Optimization, Vol. 57, pp. 395-418, 2008.

[45] H. Sun and H. Xu, Asymptotic convergence analysis for distributional robust optimization and equilibrium problems, Math. Oper. Res., to appear, 2015.

[46] F. Szidarovszky and S. Yakowitz, A new proof of the existence and uniqueness of the cournot equilibrium, Intern. Economic Rev., Vol. 18, pp. 787-789, 1977.

[47] W. Wiesemann, D. Kuhn, B. Rustem, Robust Markov decision process. Math. Oper. Res., Vol. 38, pp. 153-183, 2013.

[48] W. Wiesemann, D. Kuhn, M. Sim, Distributionally robust convex optimization. Oper. Res., Vol. 62, pp. 1358-1376, 2014.

[49] L. Xin, D.A. Goldberg and A. Shapiro, Distributionally robust multistage inventory models with moment constraints, manuscript, 2013.

[50] H. Xu, Y. Liu and H. Sun, Distributionally robust optimization with matrix moment constraints: lagrange duality and cutting plane methods, Manuscript, 2015.

[51] H. Xu and D. Zhang, Stochastic Nash equilibrium problems: sample average approximation and applications, Comput. Optim. Appl., Vol. 55, pp. 597-645, 2013.

[52] D.B. Yoffie, J. Alcacer, and R. Kim. HTC Corp. in 2012. HBS case No. 712423. Harvard Business Publishing, Boston, MA, 2012.

[53] D. Zhang, H. Xu and Y. Wu, Single and multi-period optimal inventory control models with risk-averse constraints, Eur. J. Oper. Res., Vol. 199, pp. 420-434, 2009.

[54] J. Zhang, H. Xu and L.W. Zhang, Quantitative stability analysis for distributionally robust optimization With moment constraints, Optimization-online, September 2015. 